\title{
Hitting a moving target: Perception and action in the timing of rapid interceptions
}

\author{
JAMES R. TRESILIAN \\ University of Queensland, St. Lucia, Queensland, Australia
}

\begin{abstract}
Different interceptive tasks and modes of interception (hitting or capturing) do not necessarily involve similar control processes. Control based on preprogramming of movement parameters is possible for actions with brief movement times but is now widely rejected; continuous perceptuomotor control models are preferred for all types of interception. The rejection of preprogrammed control and acceptance of continuous control is evaluated for the timing of rapidly executed, manual hitting actions. It is shown that a preprogrammed control model is capable of providing a convincing account of observed behavior patterns that avoids many of the arguments that have been raised against it. Prominent continuous perceptual control models are analyzed within a common framework and are shown to be interpretable as feedback control strategies. Although these models can explain observations of on-line adjustments to movement, they offer only post hoc explanations for observed behavior patterns in hitting tasks and are not directly supported by data. It is proposed that rapid manual hitting tasks make up a class of interceptions for which a preprogrammed strategy is adopted-a strategy that minimizes the role of visual feedback. Such a strategy is effective when the task demands a high degree of temporal accuracy.
\end{abstract}

Interactions with the environment normally occur in a dynamic context. Animals and people move through environments that themselves contain moving objects: Animals and vehicles move, and objects may fall under gravity, be blown by the wind, or thrown. A person may want to avoid colliding with a moving object or may want to bring about a controlled collision between the object and some body part or tool-that is, make an interception. A variety of ways of making an interception are possible, and these fall into two broad classes: those in which the target object is captured and those in which the target is contacted but not captured. Almost all noncapture interceptions will involve hitting.

For an interception to occur, it is necessary that the target object and the intercepting effector be at the same location (spatial coincidence) at the same time (temporal coincidence). Thus, for a particular interception location, the temporal coincidence condition can be stated as follows: The time remaining before the intercepting effector reaches the interception location (its time to arrival, TTA) must be less than or equal to the target's TTA with that location.

The intercepting effector's $T T A$ will be denoted $T T A_{\text {eff; }}$; the target's TTA will be referred to as its time to contact $\left(T T C_{\mathrm{tg}}\right)$, for consistency with other literature. In this no-

Preparation of this article was supported by a grant from the Australian Research Council. Very helpful comments from three anonymous referees are gratefully acknowledged. Correspondence concerning this article should be addressed to J. R. Tresilian, Perception and Motor Systems Laboratory, School of Human Movement Studies, University of Queensland, St. Lucia 4072, QSLD, Australia (e-mail: jamest@hms.uq.edu.au). tation, the temporal condition is that $T T A_{\text {eff }} \leq T T C_{\text {tgt }}$ (both times are positive). If $T T A_{\text {eff }}$ is less than $T T C_{\text {tgt }}$, the effector must stop and wait. The stop and wait strategy cannot be used for hitting a moving target, which requires the intercepting effector to be moving at the moment of contact. Thus, the temporal condition for hitting is that $T T A_{\text {eff }}=T T C_{\mathrm{tgt}}$. There will be some room for error, depending on the temporal accuracy constraints of the task (see Senot, Prevost, \& McIntyre, 2003; Tresilian \& Lonergan, 2002), as will be detailed later.

Despite a relatively large body of experimental and theoretical literature, there seems to be no general consensus concerning how temporal control of interception is organized. Several models have been proposed, but none is universally accepted. These models fall into two broad classes, depending on the role of exteroceptive ${ }^{1}$ information (normally derived from vision): (1) preprogrammed control models, in which movement timing is predetermined and is not influenced by exteroceptive sensory information after the motor pattern generator (the MPG) has begun to issue descending motor commands, and (2) on-line control models, in which movement timing is influenced by exteroceptive sensory information after the MPG has begun to issue descending commands. This article reviews and analyzes those models relevant to the timing of manual hitting actions. The overall scope and aims of the analysis will be detailed next.

\section{Scope: Different Tasks and Different Strategies}

The type of movements made when a target is intercepted can vary considerably, depending on the identity of the target, how it is moving, and whether it is to be captured or hit. Some interceptions require translating 
the whole body over a significant distance: running to catch a fly ball in a baseball game, a predator chasing a prey animal, or running to intercept a pass in a soccer game. In these situations, the performer will typically move to a position close to the target and then initiate a second action to contact the target-reach to catch the ball, leap onto the prey, or kick the ball.

Control of the locomotor phase has been studied and modeled for such tasks as catching projectiles (McBeath, Shaffer, \& Kaiser, 1995; McLeod \& Dienes, 1996; Michaels \& Oudejans, 1992; Tresilian, 1995) and intercepting objects moving over a ground plane (e.g., Chardenon, Montagne, Buekers, \& Laurent, 2002; Cutting, Vishton, \& Braren, 1995). All the published investigations have implicated the on-line use of visual information in locomotor guidance, but most recent models have been based on control strategies that do not involve information about where or when interception will occur. To paraphrase McLeod and Dienes (1996), the performer does not know where to go, only how to get there. These models are not appropriate for the control of arm movements when hitting or catching (McLeod \& Dienes, 1996; Tresilian, 1995). Thus, it is accepted that different interceptive tasks can involve different control strategies: Locomotor interceptions are controlled differently from manual interceptions. Only manual interceptions will be considered in what follows.

In a manual interception task, the target and the intercepting effector can approach one another from any direction, at least in principle. Figures $1 \mathrm{~A}-1 \mathrm{D}$ identify four basic approach configurations. In panel $A$, the effector pursues the target, with $V_{\text {eff }}>V_{\text {tgt }}$. In panel B, the target

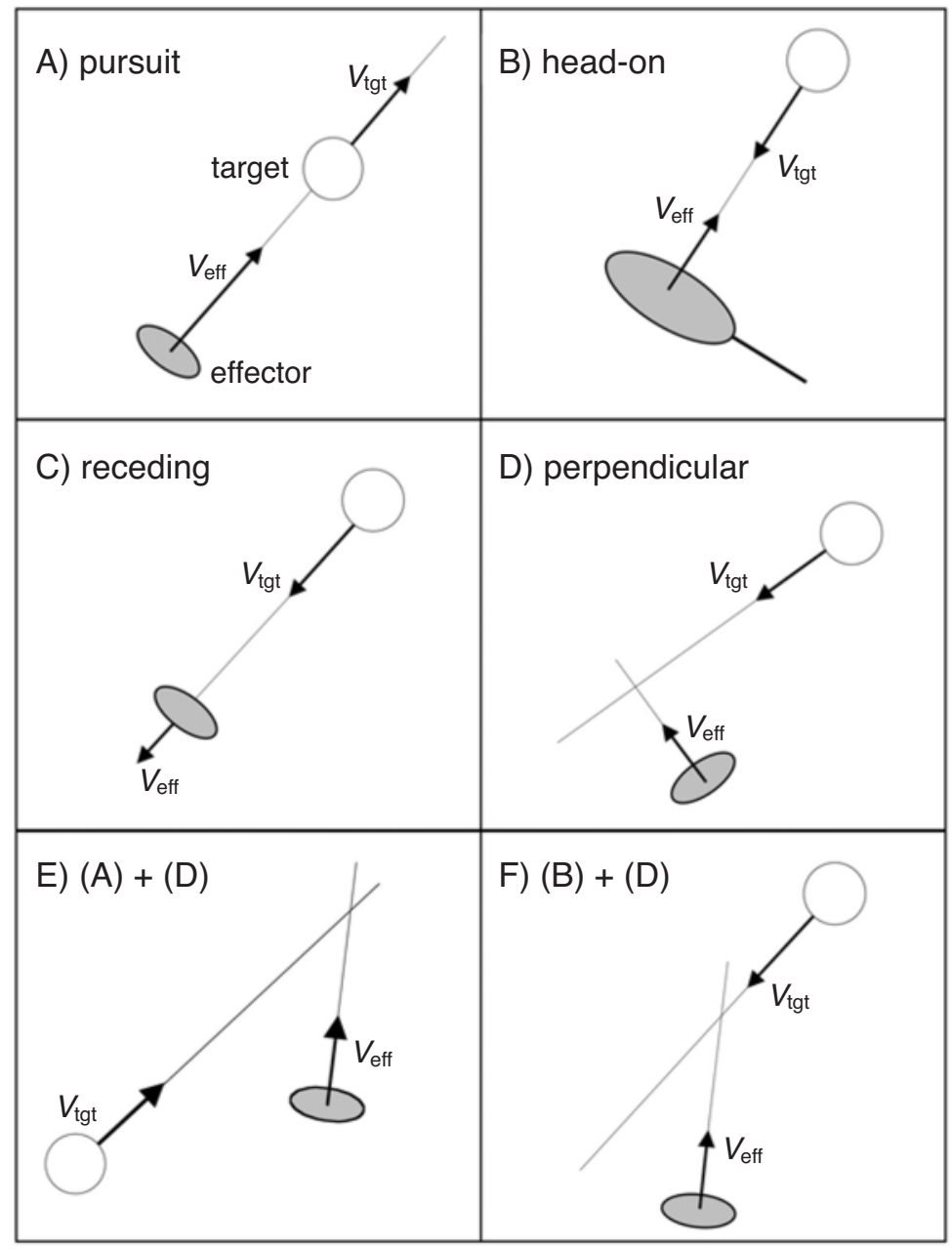

Figure 1. Simple linear approach configurations for interception. (A) Pure pursuit configuration in which the target and the intercepting effector move along the same path in the same direction. (B) Head-on collision configuration. (C) Receding configuration in which the target heads toward a slower moving effector. (D) Perpendicular approach configuration. (E) Vector combination of pursuit and perpendicular configurations. (F) Combination of head-on and perpendicular configurations. 
and the effector are on a head-on collision course. In panel C, the effector recedes from the target, with $V_{\text {tgt }}>$

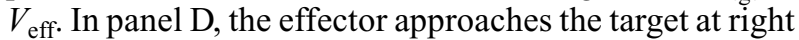
angles. Panels E and F of Figure 1 show two further configurations. In panel $\mathrm{E}$, the effector approaches the target at an acute angle. This approach can be considered a vector combination of the configurations shown in panels $\mathrm{A}$ and D. Of course, other combinations of the configurations shown in panels A-D are possible: In panel F, the approach is a vector combination of the configurations shown in panels B and D.

Manual interceptive tasks with capture can involve any of the configurations shown in Figure 1. Although capture can be executed in the pursuit (panel A) and receding (panel C) configurations, they are ill suited for hitting. Jagacinski, Repperger, Ward, and Moran (1980) reported the results of an experiment involving the pure pursuit configuration (Figure 1A). Participants manipulated a joystick (rotated it to the left or the right about a fixed axis) to move a viewing window from rest across a display screen in order to bring a moving target within the viewing window (capture task). Jagacinski et al. found that movement time (MT) increased with target speed. This is the opposite of results obtained using hitting tasks, where $M T$ has been found to decrease with increasing target speed (reviewed below). This difference suggests that capture tasks in which approach has a pursuit configuration component (e.g., Figures 1A and 1E) may involve a different control strategy than that used in hitting tasks.

In hitting tasks, it is widely assumed that movement control involves distinct and explicit representations of where to go (the interception location) and how long to take to get there (TTA; Bootsma \& van Wieringen, 1990; D. N. Lee, 1980; Regan, 1992). Models in which both time and position information are explicitly represented will be referred to as separable. The models of locomotor control described above are inseparable, since time and position are not explicitly represented. The review and analysis that follows is restricted largely to hitting tasks and to separable models that allow timing to be considered somewhat independently of positioning.

\footnotetext{
Aims

A major aim is to reconsider preprogrammed control as an account of how people time manual hitting actionsactions typically executed rapidly $(M T \mathrm{~s}<500 \mathrm{msec}$; e.g., Bootsma \& van Wieringen, 1990; Brenner, Smeets, \& De Lussanet, 1998; Watts \& Bahill, 1990). In recent years, preprogrammed control has been widely rejected as an account of rapid interceptive actions; continuous, on-line control models have been preferred (Bootsma, Fayt, Zaal, \& Laurent, 1997; Dessing, Bullock, Peper, \& Beek, 2002). In the next section, the ability of preprogrammed control to account for observed behavior will be examined, and the counterarguments will be evaluated. A similar analysis of continuous control will follow. Finally, the role of preprogrammed control in the timing of rapid, manual hitting actions will be reconsidered.
}

\section{PREPROGRAMMED CONTROL OF TIMING}

Existing preprogrammed models are all separable: They assume distinct and explicit representations of time and location information. In these models, timing control has two components: determination of the $M T$ (defined as the duration of movement from onset to target contact) and initiation of the MPG at the right moment (Fitch \& Turvey, 1978; D. N. Lee, 1980; Tyldesley \& Whiting, 1975). Exactly how the timing component of preprogrammed control allows the temporal condition for hitting to be met will be explained next.

\section{Meeting the Temporal Condition for Hitting by Preprogramming $M T$}

As was mentioned above, preprogrammed timing control involves producing a movement with a predetermined $M T$. If the programmed $M T$ takes the value $M T_{\text {prog }}$, then at the moment of movement initiation, $T T A_{\text {eff }}$ is simply $M T_{\text {prog }}$. Thus, to meet the temporal condition for hitting, the movement must start when $T T C_{\text {tgt }}=M T_{\text {prog. }}$. To achieve this, the MPG must begin to issue the descending motor command at a slightly longer $T T C_{\text {tgt }}$, to accommodate the time taken to transmit the command to the muscles and for the muscles to begin contracting in response to the command (the transmission time, TT). Thus, the MPG should begin to issue commands at a $T T C_{\text {tgt }}$ equal to $M T_{\text {prog }}+T T$. Since it takes some time for information in the retinal images to be transmitted through the nervous system to the circuits that embody the MPG (the perceptual transmission time, $P T$ ), the value of $T T C_{\mathrm{tgt}}$ at which the MPG is triggered into issuing commands should be equal to $M T_{\text {prog }}+T T+P T$. This critical value of $T T C_{\text {tgt }}$ will be written $T T C_{\text {crit. }}$ This type of scheme was termed operational timing by Tyldesley and Whiting (1975) and is a development of earlier work by Poulton $(1950,1957)$. There are two versions of operational timing in the literature, as will be described next.

\section{Two Versions of Operational Timing}

D. N. Lee (1980; see also Fitch \& Turvey, 1978) proposed a simple version of operational timing in which $M T$ was assumed fixed for a particular task. If $M T$ is invariant, the critical value of the initiating TTC information should also be invariant. In Lee's (1980) scheme, $T T C_{\text {tgt }}$ information is provided by an optic variable called $\operatorname{tau}(\tau)$. The critical value of $\tau$ used to initiate movement is assumed to be invariant for a particular interceptive task (Lee, 1980), as can be expected. As evidence for this idea, Lee (1980) cited a study of baseball batting reported by Hubbard and Seng (1954), showing that the swing times of skilled batters had little variability over repeated hits in similar conditions $(S D<20 \mathrm{msec}$, between about $5 \%-10 \%$ of the swing time). A more controlled study of batting in which computer-simulated balls were used failed to show evidence for invariant MTs (Gray, 2002a). Gray's results are consistent with a body of data demonstrating that $M T$ in 
interceptive tasks is not invariant but varies systematically with task conditions (see below). Thus, Lee's (1980) version of operational timing cannot be correct.

The existing data are compatible only with a second, more flexible version of operational timing, in which $M T$ can be preprogrammed to take different values. The preprogramming process must also determine the critical value of the $T T C_{\mathrm{tgt}}$ information used for initiation, since $T T C_{\text {crit }}=M T_{\text {prog }}+T T+P T$ (i.e., $T T C_{\text {crit }}$ depends on $\left.M T_{\text {prog }}\right)$. A block diagram illustrating the functional components of this version of operational timing is shown in Figure 2A; Figure 2B shows the temporal sequence of events. Three basic questions can be asked about the hypothetical $M T$ preprogramming process. (1) What factors determine the value of the programmed MT? (2) When does the programming process start and finish, and when are the different factors integrated into the program? (3) What factors affect the success of the action? These three questions will be addressed in what follows.

\section{What Factors Influence Movement Time?}

It is clear from watching people play ball sports that they can choose whether to move slowly and hit a target softly (longer $M T$ ) or to move quickly and hit it hard (shorter $M T$ ). This ability can be studied experimentally by instructing people to hit hard or soft or by setting a goal outcome that is dependent on the strength of the hit. Caljouw, van der Kamp, and Savelsberg (2004) recently reported some experiments that required making harder or softer hits; the participants were able to meet these task requirements successfully. Beyond this, there has been little experimental study of this ability.

The strategy in most research has been to vary the task conditions and observe how performance variables such as $M T$ change in response. It has been found that $M T$ and/or maximum movement speed vary systematically with changes in task parameters, such as target speed and the distance moved to reach the target (Bairstow, 1987; Brenner et al., 1998; Gray, 2002a; Schmidt, 1969; van Donkelaar, Lee, \& Gellman, 1992; Zaal, Bootsma, \& van Wieringen, 1999). A consistent finding has been that people make briefer and/or faster movements when the target moves more quickly, but this result has been difficult to interpret, since target speed is typically confounded with viewing time ( $V T$; Ball \& Glencross, 1985; Mason \& Carnahan, 1999). VT is the time for which the target can be seen prior to contact ( $=Z / V$ in Figure 3 ; see below). If $V T$ and $V$ covary, it is impossible to determine whether the effects on performance are due to changing $V$ or changing VT (Mason \& Carnahan, 1999). With a shorter

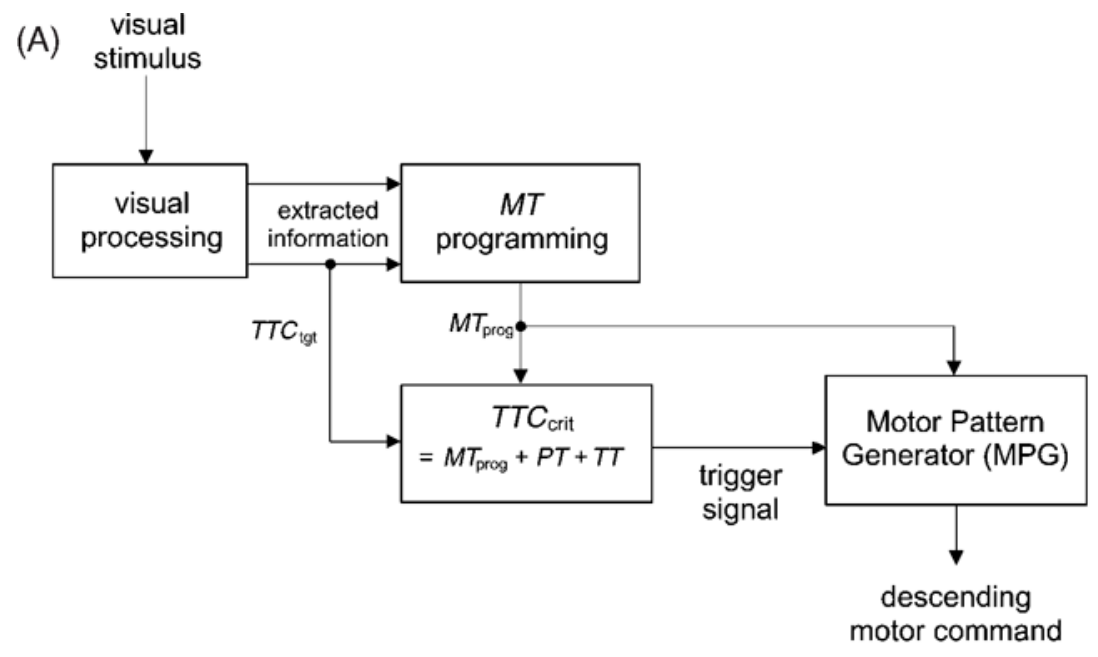

(B) $M T$ preprogrammed

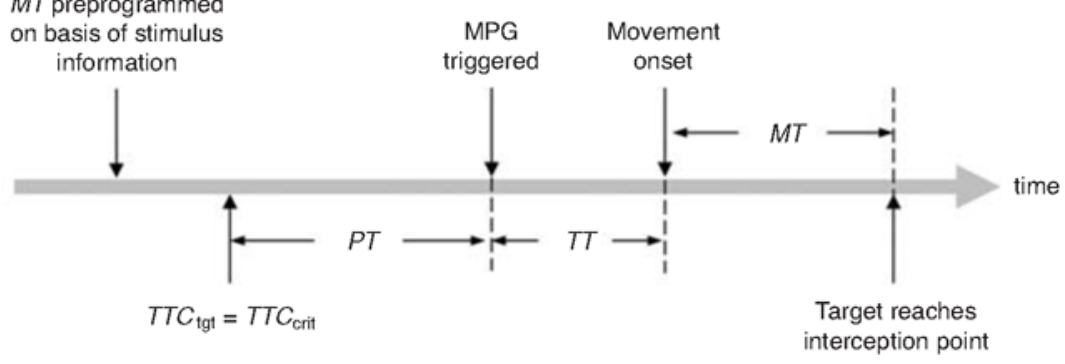

Figure 2. (A) Functional block diagram of the operational timing scheme with programmable movement time (MT) described in the text. (B) Temporal sequence of events involved in the scheme. $T T C$, time to contact; $P T$, perceptual transmission time; $T T$, transmission time. 


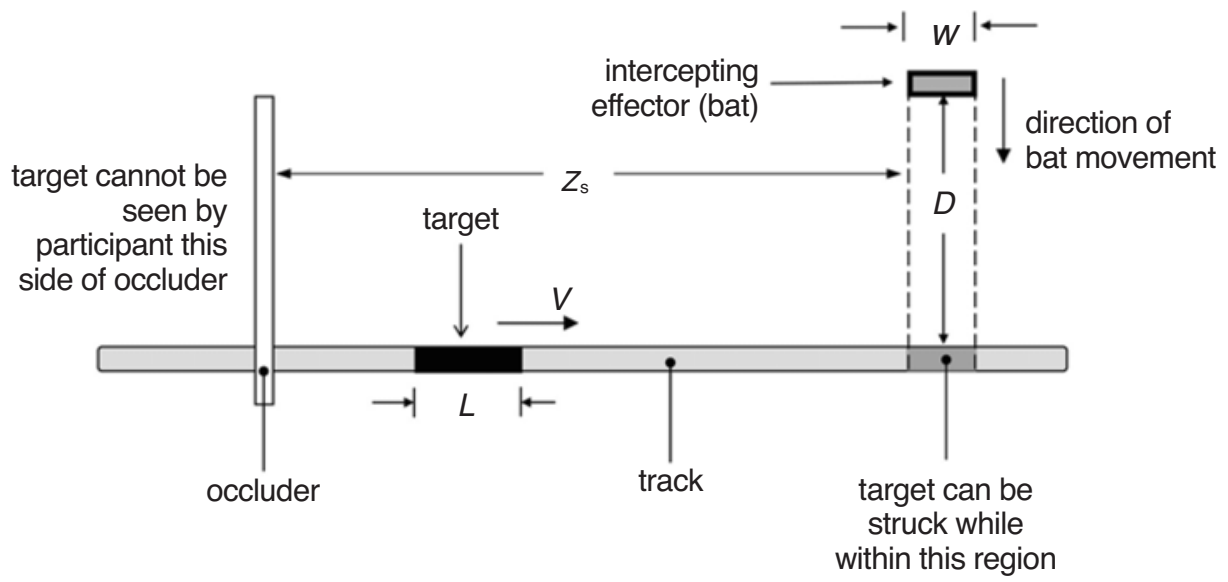

\begin{abstract}
Figure 3. The one degree of freedom hitting task. The intercepting effector (bat of width $W$ ) is constrained to move along a straight path perpendicular to the path of the target. The target moves at a speed $V$ and has a length $L$ along its direction of travel. The bat must move a distance $D$ to reach the target. The target is visible to the performer from a distance $Z_{\mathrm{s}}$ from the interception location.
\end{abstract}

$V T$, there is less time to see the target and execute the response: Briefer, faster movements are, therefore, to be expected, and this has been confirmed in experiments (Laurent, Montagne, \& Savelsbergh, 1994; Mason \& Carnahan, 1999; Montagne, Fraisse, Ripoll, \& Laurent, 2000).

Figure 3 illustrates a simple hitting task in which the $V T$ and target speed $(V)$ can be manipulated independently by changing the viewing distance $(Z)$. To fix the distance $Z$ and, hence, the $V T$, it is necessary that the person performing the task cannot choose where they will intercept the target. In this task, the performer is constrained to strike the target by moving a handheld manipulandum along a fixed, straight track perpendicular to the target's path (approach configuration shown in Figure 1D). Since movement has only one spatial degree of freedom $(d f)$, the task is a $1-d f$ hitting task.

The advantage of the $1-d f$ task's approach configuration is that both $V T$ and the required temporal accuracy can be controlled (this is not possible using the approach configurations shown in Figures 1A-1C). The temporal accuracy demand is quantified by the time window- the period of time for which the target is within the strike zone (Tresilian \& Lonergan, 2002). The time window is given by $(L+W) / V$, where $L$ is the target's length, $W$ is the manipulandum width, and $V$ is the target's speed (Figure 3 ).

Using the $1-d f$ task, we have found that when $V T$ is held constant, $M T$ increases in approximate proportion to the time window and the distance moved ( $D$ in Figure 3 ); it also decreases with increasing target speed independently of the time window (Tresilian \& Houseman, in press; Tresilian \& Lonergan, 2002; Tresilian, Oliver \& Carroll, 2003). These results can be summarized by the following statement relating $M T$ to the independent variables:

$$
M T=a+b D-c V+d[L+W] / V,
$$

where $a-d$ are empirical parameters (Tresilian \& Houseman, in press). Results from other rapid interception tasks have shown similar effects of $D$ (Schmidt, 1969; Zaal et al., 1999) and $V$ (Brouwer, Brenner, \& Smeets, 2000; Gray, 2002a).

The results summarized by Equation 1 were obtained when $V T$ was maintained at a value that was relatively large (between 1.3 and $1.7 \mathrm{sec}$, depending on the experiment), as compared with the range of $M T$ s obtained (from about 80 to $400 \mathrm{msec}$ ). Mason and Carnahan (1999) reported the absence of a statistically reliable effect of target speed on $M T$ with a constant $V T$ of $1 \mathrm{sec}$ and $M T \mathrm{~s}$ of about $700 \mathrm{msec}$ (on average) in a catching task. Clearly, in our experiments, the participants had much more time to view the target prior to starting the movement (on average, about $1.2 \mathrm{sec}$ ), as compared with the participants in Mason and Carnahan's study (about $300 \mathrm{msec}$ ). Taken together, these results suggest that $V T$ and target speed interact in determining $M T$ such that when the $V T$ is sufficiently short, the person has too little time available to vary $M T$ in response to changes in target speed (or the other parameters in Equation 1). In a recent experiment, we investigated this possibility by examining the effect of target speed on $M T$ for five different $V T$ s between 400 and $1,000 \mathrm{msec}$, using the $1-d f$ task (Tresilian \& Houseman, in press). It was found that both the slope of the $M T$-target-speed relationship and its intercept with the $M T$ axis decreased with decreasing $V T$, the slope being indistinguishable from zero at the 400 -msec $V T$. This result confirmed the expectation and can be interpreted as meaning that some of the parameters $(a-d)$ that appear in Equation 1 depend on the $V T$.

Finally, it should be noted that if $V T$ is held constant over changes in $V$, viewing distance $(Z)$ must be changed, since $V T=Z / V$. It appears, however, that performance is affected primarily by $V T$ and $V$, since empirical studies have failed to show an independent effect of viewing distance (Ball \& Glencross, 1985; Fleury, Basset, Bard, \& Teasdale, 1998; Montagne et al., 2000). 


\section{The Programming Process}

According to the second version of operational timing (Figure 2), the systematic effects on $M T$ described by Equation 1 are due to programming different $M T$ s for different task conditions. Thus, Equation 1 might directly reflect the rule used to preprogram $M T$ (Tresilian, 2004): The $M T$ programming process uses information about the task variables $(D, L, W$, and $V)$ to determine $M T$, using a rule of the form of Equation 1, with $V T$ determining the value of some or all of the parameters.

In principle, the $M T$ programming process could take place within the $V T$ period. However, this seems unlikely, especially when the $V T$ is very short (e.g., $<500 \mathrm{msec}$ in baseball). It is more plausible to suggest that people prepare themselves in advance of seeing the target on the basis of their expectations. These establish a state of preparedness, or motor set, in which MT and other movement parameters are imprecisely specified. During the $V T$, they fine-tune the prepared $M T$ on the basis of the information that then becomes available, but within the constraints imposed by the motor set. In our experiments, the motor set might be based on information available prior to the person's seeing the target, which would include the distance to be moved $(D)$ and the width of the intercepting effector $(W)$. In addition, it would likely depend on the person's experience with the task: As a person becomes familiar with the task over repeated trials, he or she learns about the range of target speeds, target sizes, and $V T \mathrm{~s}$.

Preparedness based on advance information is an established concept in human motor control (see, e.g., Schmidt, 1988) and is likely to play a significant role in interception, as is suggested by the results reported by De Lussanet (2001; De Lussanet, Smeets, \& Brenner, 2001) and Gray (2002a, 2002b). Both De Lussanet and Gray found that performance on a particular experimental trial was systematically influenced by what the participant had experienced on the immediately preceding trials. Gray (2002a) found that when the speed of a to-beintercepted target varied randomly over a relatively wide range, interception performance was significantly worse than when speed could vary only over a much smaller range. These kinds of results are consistent with the observations of sportspeople (e.g., Williams \& Underwood, 1970) and with results reported in the sports psychology literature suggesting that advance information about the likely trajectory of a target is important for good performance (Abernethy, 1990).

\section{What Factors Affect the Success of an Attempted Interception?}

Successful timing requires the following: (1) initiation of the movement at the right moment in time (when $\left.T T C_{\text {tgt }}=M T_{\text {prog }}\right)$, (2) accurate detection of the moment when $T T C_{\mathrm{tgt}}=T T C_{\text {crit }}$, and (3) execution of the movement in the predetermined time $\left(M T_{\text {prog }}\right)$. The ability to meet these requirements will be limited by internal noise in the sensory and motor systems. Noise in the sensory systems introduces uncertainty into the determination of when $T T C_{\text {tgt }}=T T C_{\text {crit }}$; noise in the motor system will result in the actual $M T$ being different from $M T_{\text {prog. }}$. External disturbances to movement (external noise) will also compromise the ability to produce an $M T$ that matches $M T_{\text {prog }}$, and any errors that result are uncorrected (no feedback control; see the On-Line Control of Timing section).

The ability to start moving when $T T C_{\text {tgt }}=M T_{\text {prog }}$ will also be influenced by the accuracy of the TTC information used for initiation. For example, Lee's (1980) suggestion that $\tau$ is used for movement initiation will lead to timing errors, since $\tau$ provides only an approximation to the true TTC. Since I have discussed this matter extensively elsewhere (Tresilian, 1994a), it will not be considered further here. However, it has recently been suggested that the initiating variable is not a source of TTC information per se but, rather, the rate of expansion $(R O E)$ of the target's image (Michaels, Zeinstra, \& Oudejans, 2001; Smith, Flach, Dittman, \& Stanard, 2001). Whether movement initiation based on the $R O E$ is compatible with operational timing will be considered later.

The ability to produce a movement of preprogrammed duration depends on the peripheral neuromechanical ${ }^{2}$ system responding appropriately to the commands issued by the MPG. The aim is to produce a movement pattern that has the required characteristics $(M T)$, and this can be done only if the commands are correct for the neuromechanics being controlled. Thus, generation of the correct commands requires some kind of knowledge of the neuromechanical system-knowledge that is typically conceived to be some kind of internal model of that system (e.g., Kawato, 1999). The ability to make a movement of a precise duration (MT) requires an internal model that itself has sufficient precision; imprecision in the model will yield imprecision of movement.

Finally, although movement timing and positioning may be separable, they are, nevertheless, intimately connected. In an interceptive task in which a person is attempting to move a specific distance in a specific time, a temporal error can also be represented as a spatial error (Newell, 1980). Thus, a person can arrive at the interception location in the $1-d f$ task (Figure 3 ) too early either by moving the correct distance in too short a time or by moving too far in the right time. Some implications of this will be returned to later.

\section{Optimizing Performance in the Presence of Noise: Brevity Improves Timing}

The perturbing effects of noise, both internal and external (unanticipated disturbances), will have less effect on timing for shorter $M T \mathrm{~s}$, since there is less time for the effects of motor noise ${ }^{3}$ to accrue (Meyer, Kornblum, Abrams, Wright, \& Smith, 1988; Schmidt, Zelaznik, Hawkins, Frank, \& Quinn, 1979) and less opportunity for external disturbances to affect movement. Consistent with this, in movement tasks in which the aim is to complete a movement directed at a stationary target in an experimenter-specified time (the required $M T$ ), $M T$ is less variable (more reproducible) when the required $M T$ 
is short (Newell, Hoshizaki, Carlton, \& Halbert, 1979; Schmidt et al., 1979). Thus, it might be expected that when a person needs to execute a movement that is functionally complete at a precise moment (such as an interceptive movement), he or she will choose to make a briefer movement (Schmidt, 1988). Our experiments with the $1-d f$ task confirm this expectation: When people needed to be more temporally accurate (smaller time window), their MTs were shorter (Equation 1), and they were more temporally accurate (Tresilian et al., 2003). Note that it is still necessary to explain why people move more slowly when less temporal precision is required. Why not always make a brief movement? A plausible reason relates to the fact that rapid movements require more effort (greater muscular forces) than do slower movements over the same distance: People prefer to make movements that require less effort (see, e.g., Alexander, 1997; Sparrow, 2000).

Briefer movements not only reduce the disturbing effects of motor and external noise, but also may do the same for sensory noise. Visual estimation of TTC appears to obey a Weber's law type relationship, with $T T C$ discrimination thresholds increasing with the magnitude of TTC (Gray \& Regan, 1998; Regan \& Hamstra, 1993), implying a multiplicative noise process (Green \& Swets, 1966). Thus, the smaller the value of $T T C_{\text {crit }}$ in operational timing (Figure 3), the better it can be estimated and the moment of movement initiation more precisely determined. Gray and Regan (1998) showed that human observers are capable of very accurate single estimates of TTC that are within $\pm 5 \%$ of the true value, sometimes within as little as $\pm 2 \%$. Even at $500 \mathrm{msec}$ before contact, a $2 \%$ error in $T T C$ estimation is only $10 \mathrm{msec}$.

Another benefit of making briefer movements is that given a particular $V T$, shorter $M T$ s allow the person to view the target for longer prior to initiating the movement (Breen, 1967; Schmidt, 1988). Viewing the target for longer, even only 50-100 msec longer, has been found to improve performance in some interceptive actions (Elliott, Zuberec, \& Milgram, 1994; Sharp \& Whiting, $1974,1975)$. The strategy of extending the $V T$ by shortening the $M T$ may help explain why target speed had an effect on $M T$ additional to its effect via the time window (Equation 1). When the target moves more quickly, it is more difficult to pursue it with the eyes and keep its image foveated (Carpenter, 1977). Thus, it is possible that the additional effect of target speed may reflect the strategy of delaying the action until sufficiently accurate perceptual estimates of the necessary informational quantities have been obtained.

\section{Rapid Movements Reduce the Impact of Spatial (Amplitude) Errors}

Bootsma and van Wieringen (1990) studied skilled table tennis players and concluded that operational timing could not explain their performance, since unless contact could be guaranteed to occur at exactly the right location, timing would be inaccurate. Consider as an ex- ample the $1-d f$ hitting task (Figure 3 ). If we imagine that the performer is attempting to strike the center of the target with the middle of the manipulandum, it is possible to define a temporal error. ${ }^{4}$ The temporal error is the period of time between the moment the center of the target is in line with the path of the middle of the manipulandum $\left(t_{\mathrm{d}}\right)$ and the moment $\left(t_{\mathrm{c}}\right)$ the middle of the manipulandum reaches the target (i.e., has traveled a distance $D$; Figure 3 ). If the magnitude of the temporal error is greater than half the time window, the person misses the target. As was noted earlier, a temporal error can also be represented as a spatial error in an interceptive task such as the $1-d f$ task. The distance of the middle of the manipulandum from the target's center at time $t_{\mathrm{d}}$ is the spatial error. The fact that temporal errors can be expressed as spatial errors and vice versa means that misprogramming of the distance or the duration of a movement in the $1-d f$ task will have equivalent effects on performance-it is impossible to tell whether the person has misprogrammed distance or $M T$ by observing performance errors. However, we can answer the following question: How large are the spatial errors associated with temporal errors of $\pm 1 / 2$ (time window)? Or equivalently, how large can the error in programming movement distance be if the timing error is zero?

In our $1-d f$ experiments, the participants struck the targets close to the moment of peak velocity (as in other experiments; see, e.g., Bootsma \& van Wieringen, 1990; Montagne et al., 2000). Peak velocities typically exceeded $2 \mathrm{~m} / \mathrm{sec}$ and were sometimes in excess of $5 \mathrm{~m} / \mathrm{sec}$; in baseball, peak bat speeds in excess of $35 \mathrm{~m} / \mathrm{sec}$ are common (Welch, Banks, Cook, \& Draovitch, 1995). Consider hitting a target with a $60-\mathrm{msec}$ time window with movements such as those shown in Figure 4 (panel A, position; panel $\mathrm{B}$, speed). One movement is programmed and executed perfectly: The manipulandum strikes the center of the target at the moment of peak velocity (solid curves, Figure 4). Also shown are two perfectly timed movements whose distances have been misprogrammed: One is too short (dotted curve), and one is too long (dashed curve). The shorter movement reaches a slightly lower peak speed than the perfectly executed movement (Figure 4B), and the longer one reaches a slightly higher peak speed. If the short movement is to strike the target, the distance error must be made up within $30 \mathrm{msec}$ of peak speed. If the average speed over a $30-\mathrm{msec}$ period following peak speed is $V \mathrm{~cm} / \mathrm{sec}$, the distance traveled in that time is $0.03 \mathrm{~V} \mathrm{~cm}$. Thus, for average speeds of 1 and $2 \mathrm{~m} / \mathrm{sec}$, the spatial errors will be 3 and $6 \mathrm{~cm}$, respectively-more than $20 \%$ of movement distances between 10 and $40 \mathrm{~cm}$ (the range in our $1-d f$ experiments). For faster movements, even larger amplitude errors can be accommodated. Thus, the amplitude of very rapid interceptions does not necessarily need to be precisely programmed in an operational timing scheme. The direction of the movement may need to be more accurately preprogrammed, as Bootsma and van Wieringen (1990) suggested. However, the directional accuracy and preci- 

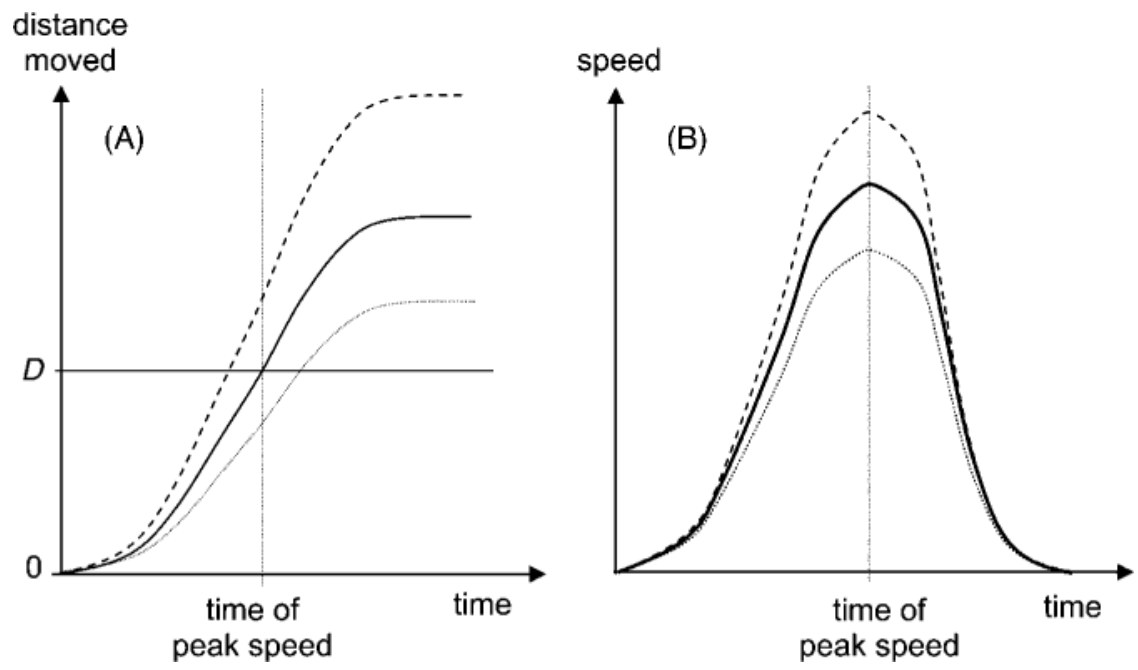

Figure 4. (A) Position and (B) velocity profiles of three hypothetical hitting movements made in attempts to hit a moving target a distance $D$ from a start position (0). Solid curves: an "ideal" hit in which the middle of the target is struck at the moment of peak velocity. Dotted and dashed curves: two accurately timed movements whose amplitudes have been misprogrammed to be too short (dotted curve) or too long (dashed), so that peak velocity is reached at the right time (same moment as the ideal movement), but at a location in front of the target (dotted) or behind the target (dashed).

sion of rapid, visually open-loop movements can be very good (Howarth \& Beggs, 1985; Schmidt \& Sherwood, 1982).

\section{Use of the ROE to Elicit a Predetermined Response}

It has recently been proposed that initiation of movement in interceptive actions is based on the $R O E$ of the approaching target's retinal image reaching a critical value that is constant over changes in the task conditions (Michaels et al., 2001; Smith et al., 2001). This predicts earlier response onsets (and hence, longer $M T \mathrm{~s}$ ) to slower moving and larger targets (see Smith et al., 2001). Smith et al. have argued that initiation based on a constant $R O E_{\text {crit }}$ does away with the need for TTC information and can provide a unified account of timing errors in some other studies that were previously interpreted in several different ways (e.g., DeLucia, 1991; DeLucia \& Warren, 1994; Sidaway, Fairweather, Sekiya, \& McNittGray, 1996). This hypothesis offers no account of how $M T$ is determined; it relates only to movement initiation. Since the moment of initiation can be earlier or later, depending on the speed and size of the target, some way of ensuring that the $M T$ is appropriate for the target's TTC is necessary (in order to meet the temporal condition for interception). This means that $M T$ must be determined from an estimate of $T T C_{\text {tgt }}$ when the $R O E$ reaches $R O E_{\text {crit }}$.

The $R O E$ hypothesis does not account for variations in $M T$ (and initiation time) with distance $(D)$, manipulandum width $(W)$, and viewing time (VT; e.g., Equation 1). To deal with these variations, it would have to be supposed that the person uses a different value of $R O E_{\text {crit }}$ for different values of $D, W$, and $V T$. Thus, to explain the available data, the idea of a fixed value of $R O E$ must be abandoned and replaced with the idea that it remains the same across some task variations (e.g., in target size and speed) but varies with others (e.g., $D, W$, and $V T$ ). This process must be completed before the MPG is triggered. The time line of events in such a scheme would be as shown in Figure 5.

These considerations show that within the operational timing framework, the original rationale for the $R O E$ hypothesis is lost: A fixed critical $R O E$ value can explain only some of the data (to account for other data, $R O E_{\text {crit }}$ must vary) and TTC information is still necessary. Similar arguments apply for any quantity other than TTC that has been proposed as the basis for movement initiation, such as distance (van Donkelaar et al., 1992) or additive combinations of image size and ROE (Smith et al., 2001).

\section{Further Limitations of Operational Timing}

There are two objections to preprogrammed control of timing that have been raised in the literature, which must be considered before operational timing can be considered a viable alternative to on-line control. First, several experiments have provided evidence for corrections or adjustments to interceptive movements during execution (Brenner et al., 1998; D. Lee, Port, \& Georgopoulos, 1997; Montagne, Laurent, Durey, \& Bootsma, 1999; van Donkelaar et al., 1992). These observations cannot be explained by preprogrammed control models, an issue that will be returned to in the Discussion section.

Second, it has been observed in hitting experiments that variability in movement onset time is greater than the variability of strike time over multiple trials in which the task conditions are the same. Bootsma and van Wieringen 


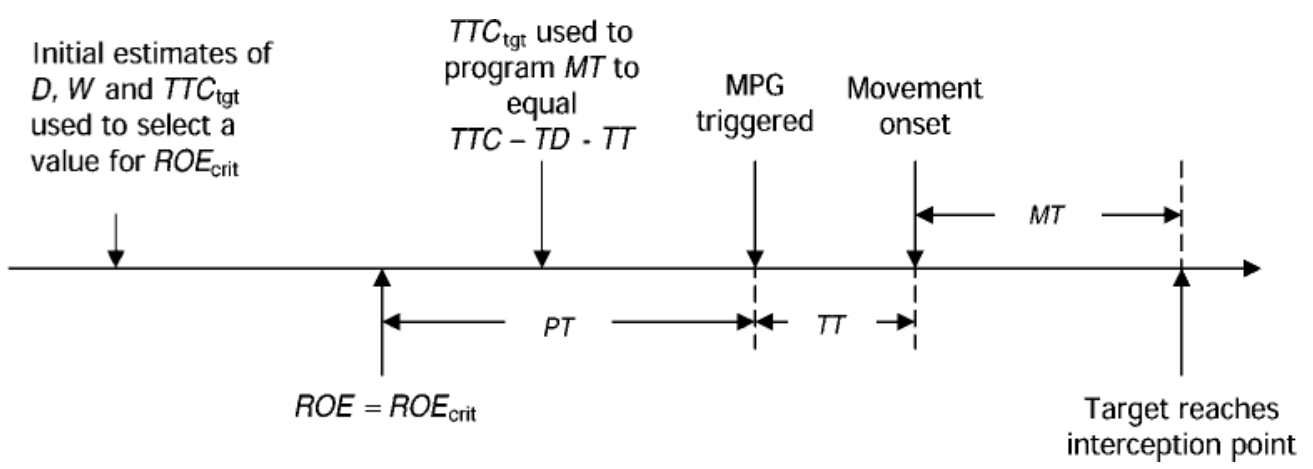
Figure 5. Time line of events in an operational timing scheme in which the rate of expansion $(R O E)$ of
the target's retinal image(s) is used to initiate the motor pattern generator (MPG).

(1990), who first reported this observation, have argued that it cannot be accounted for by an operational timing scheme. The observation was replicated in our experiments with the $1-d f$ task. $M T$ variability $(S D)$ was greater than the time window $(2 \times S D>$ time window $)$ over trials in which the target was successfully struck. Thus, variability of movement onset times was greater than the variability of strike times. The version of operational timing described here can account for this observation as follows. In constant task conditions, the programmed $M T$ can vary due to changes from trial to trial in a person's internal state (e.g., motivation or fatigue); in randomized repeated measures designs, it can also be affected by the sequential dependencies between trials reported by De Lussanet (2001) and Gray (2002a). Variability in $M T_{\text {prog }}$ will be reflected in movement onset time variability, since variations in $M T_{\text {prog }}$ cause variations in $T T C_{\text {crit }}(=$ $\left.M T_{\text {prog }}+T T+P T\right)$, and variation in $T T C_{\text {crit }}$ results in onset time variability. The effect of the variation in $T T C_{\text {crit }}$ and, hence, of onset time is to ensure that the temporal condition for hitting is met despite variations in $M T_{\text {prog }}$. In other words, onset time variability is not just noise; it compensates for variations in $M T_{\text {prog }}$ to ensure that strike time does not vary. Thus, far from being refuted by the observation that onset time variability is greater than strike time variability, the version of operational timing described here can be considered to predict this result (provided variability in $M T_{\text {prog }}$ is greater than the effects of motor noise on $M T$, since the latter will affect strike time variability, but not onset variability).

\section{Summary}

The review and analysis of operational timing presented in this section is summarized schematically in Figure 6.

\section{ON-LINE CONTROL OF TIMING}

Two types of on-line control models have been suggested for how exteroceptive visual information might influence movement timing: first, continuous control models, in which a visually derived signal forms a con- tinuous controlling input that drives movement production (Dessing et al., 2002; D. N. Lee, Young, Reddish, Lough, \& Clayton, 1983; Peper, Bootsma, Mestre, \& Bakker, 1994), and second, discrete error correction models similar in concept to those proposed for movements aimed at stationary targets (see, e.g., Elliott, Helsen, \& Chua, 2001; Meyer et al., 1988), in which an initial preprogrammed movement is corrected by one or more subsequent discrete corrective submovements produced in response to perceptually detected errors in the unfolding movement (D. Lee et al., 1997; Tyldesley \& Whiting, 1975). Of the two types, only continuous control has given rise to explicitly formulated models of interception that have been tested empirically. These models involve no preprogrammed component, and so the entire movement is driven by a perceptually derived signal. This section will focus on models of this kind.

\section{Meeting the Temporal Condition for Interception Using Continuous Control}

The temporal condition for hitting presented in the introduction can be restated as a condition that should hold at every instant during execution of a movement. In these terms, the condition reads as follows: At any moment of time during performance, the time remaining until the intercepting effector reaches the interception location ( $\left.T T A_{\text {eff }}\right)$ should be less than or equal to the time remaining before the target arrives there $\left(T T C_{\mathrm{tg}}\right)$. That is, $T T A_{\mathrm{eff}}(t) \leq$ $T T C_{\mathrm{tgt}}(t)$ at all times, $t$ [for hitting, $T T A_{\mathrm{eff}}(t)=T T C_{\mathrm{tgt}}(t)$ ].

The hitting condition is clearly satisfied when $T T A_{\text {eff }}-$ $T T C_{\mathrm{tgt}}=0$. Thus, the temporal control problem for anticipatory hitting could be viewed as that of reducing an error $\left(T T A_{\mathrm{eff}}-T T C_{\mathrm{tgt}}\right)$ to zero. In control engineering, such problems are often solved using negative feedback control, and the simplest method is to use a proportional feedback control law (see, e.g., Brogan, 1990) - that is, to generate a force, proportional to the magnitude of the error, that acts to eliminate the error. Such a control law would specify the application of a resultant force $(F)$ on the intercepting effector according to the rule

$$
F=G\left(T T A_{\text {eff }}-T T C_{\text {tgt }}\right)
$$


Vision supplies information about taskrelevant variables including $W, L, V, D$, and $T T C_{\text {tgt }}$ (Equation 1). Other sensory systems may also be involved.
Preprogrammed $M T\left(M T_{\text {prog }}\right)$ depends on:

(1) Perceptual information about task variables

(2) Internal state (e.g., motivation, fatigue)

(3) Advance information \& expectation

(2) and (3) mean that $M T_{\text {prog }}$ can vary over repeated trials in which (1) is always the same

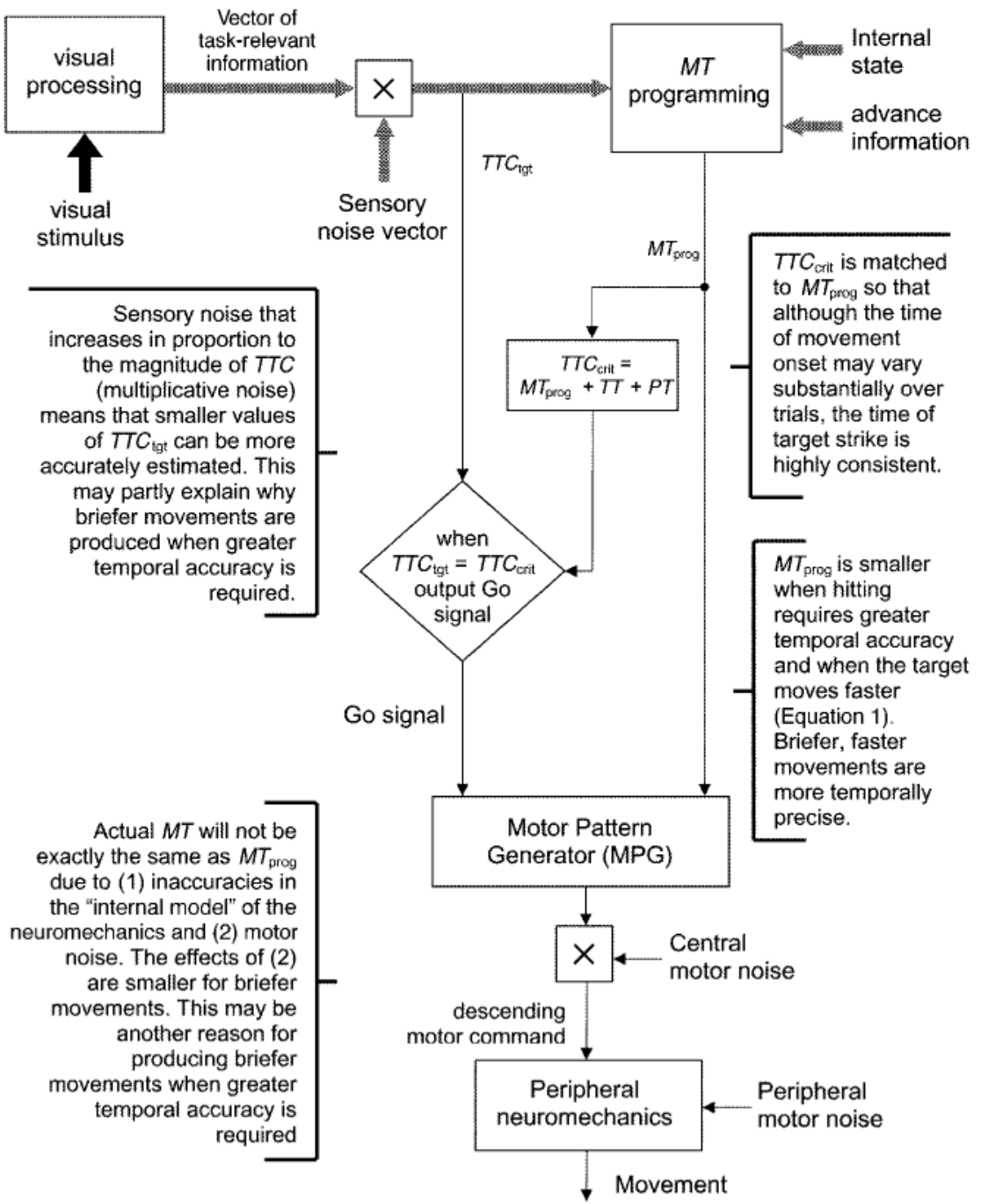

Figure 6. Summary of preprogrammed control of timing showing an extension of the block diagram of Figure $2 \mathrm{~A}$. " $\mathrm{X}$ " indicates that the sensory and central motor noise processes are multiplicative.

where $G$ is the feedback gain. Ideally, the applied force will act to progressively drive the error $\left(T T A_{\text {eff }}-T T C_{\mathrm{tgt}}\right)$ to zero. At the start of the movement, the error will be large but will become smaller and smaller.

The control law defined by Equation 2 requires perceptual measurement of the error. To do this, both $T T A_{\text {eff }}$ and $T T C_{\mathrm{tgt}}$ need to be measured. It has been proposed that people do not perceptually estimate the exact $T T A$ but, rather, a first-order approximation to the TTA defined by the current distance away divided by the speed of approach (D. N. Lee, 1998), a view supported by em- pirical data (see Senot et al., 2003; Tresilian, 1997). This approximation is often denoted $\tau(d)$, where $d$ is the distance away (D. N. Lee, 1998). If a person used first-order TTA estimates to form the error, Equation 2 would be modified to read

$$
F^{\prime}=G^{\prime}\left[\tau_{\text {eff }}-\tau_{\text {tgt }}\right]
$$

where $\tau_{\text {eff }}$ is the instantaneous distance of the effector from the interception location divided by its speed and $\tau_{\text {tgt }}$ is the same quantity defined for the target. If successfully implemented, this control law will drive the 
error $\left(\tau_{\text {eff }}-\tau_{\text {tgt }}\right)$ to zero, so the value of $\tau_{\text {eff }}$ will get closer and closer to the value of $\tau_{\text {tgt }}$. Lee (1998) calls this behavior tau-coupling but does not specify the control law: Equation 3 is a tau-coupling control law because it explicitly tries to make $\tau_{\text {eff }}$ equal to $\tau_{\text {tgt }}$. If any type of interception (not just hitting) is required, the condition $T T A_{\text {eff }}-T T C_{\text {tgt }} \leq 0$ should hold at all times. This implies that $T T A_{\text {eff }}-\beta T T C_{\text {tgt }}=0$, where $0<\beta \leq 1$ or, for tau-coupling, $\left(\tau_{\text {eff }}-\beta \tau_{\text {tgt }}\right)=0$. Thus, as collision approaches, $\tau_{\mathrm{eff}} \approx \beta \tau_{\mathrm{tgt}}$, which is Lee's original definition of tau-coupling (Lee, 1998).

\section{Required Velocity Control}

The resultant force $\left(F^{\prime}\right)$ in Equation 3 is applied to the intercepting effector, and so control is achieved by changing the velocity of the effector (a person cannot influence the motion of the target). Thus, the tau-coupling control law achieves the temporal condition for hitting (approximated as $\tau_{\text {eff }}=\tau_{\text {tgt }}$ ) by modulating the velocity of the intercepting effector. $\tau_{\text {eff }}$ is $D(t) / V(t)$, where $D(t)$ is the effector's distance from the interception location and $V(t)$ is its speed at time $t$. The temporal condition for hitting can be written $\tau_{\text {tgt }}=D(t) / V(t)$; thus, the speed of the effector will be equal to $D(t) / \tau_{\text {tgt }}$ when the condition is met. This means that the control law can be formulated in terms of the speed of the effector required to meet the temporal condition: The required speed $\left(V^{*}\right)$ is equal to $D(t) / \tau_{\text {tgt. }}$. A control law that reduces the difference between $V^{*}$ and $V$ (the actual speed of the effector) to zero will ensure accurate timing. $V^{*}-V$ is thus an error, and a suitable proportional feedback control law is

$$
F=H\left(V^{*}-V\right),
$$

where $H$ is the feedback gain. This method of control involves perceptual estimates of $V$ and $V^{*}$ (as $D[t] / \tau_{\text {tgt }}$ ) and so differs from tau-coupling, which requires estimates of $\tau_{\text {eff }}$ and $\tau_{\text {tgt }}$. The control law described by Equation 4 is an example of what has been called required velocity control (RVC; Bootsma et al., 1997; Montagne et al., 1999; Peper et al., 1994). Montagne et al. (1999; see also Bootsma et al., 1997) formulated RVC in terms of the effector's acceleration $(A)$, rather than force $(F)$. This can easily be done for Equation 4 if the end-effector to which the force $(F)$ is applied is treated as an abstract point mass $(M)$ in the manner introduced by Saltzman and Kelso (1987). This allows application of Newton's second law $(F=M A)$, and Equation 4 becomes

$$
A=(H / M)\left(V^{*}-V\right) \text {. }
$$

In Montagne et al.'s (1999) formulation, $A=m V^{*}-n V$, where $m$ and $n$ are constants. In Equation 5, the constant multiplier $(H / M)$ is the same for both $V^{*}$ and $V$. This difference derives from the fact that for interceptions in general (not just hits), the temporal condition is approximated as $\tau_{\text {eff }}=\beta \tau_{\text {tgt }}$ and can be written $\beta \tau_{\text {tgt }}=D(t) / V(t)$. This means that Equation 5 becomes $A=(H / M)\left(V^{*}-\beta V\right)$, which is equivalent to Montagne et al.'s (1999) formulation.

\section{Earlier Formulations of RVC}

Versions of RVC were first proposed independently by Peper et al. (1994) and Tresilian (1994b) in two rather different formulations, both of which differ from Equations 4 and 5. Peper et al. assumed that the interception location is never accurately estimated but is converged upon as movement evolves (see also Bootsma et al., 1997). Thus, the performer is assumed never to directly estimate the distance to the interception location $(D$ in Figure 7). Instead, he or she makes a continuous estimate of the distance between the intercepting effector and the lateral position of the moving target, $X(t)$ in Figure 7. The required velocity $\left(V^{*}\right)$ is then determined as $X(t) / \tau_{\text {tgt }}(t)$, where $\tau_{\text {tgt }}$ is the tau-function of the distance $Z$ ( $=$ the target's TTA with the interception point when $\mathrm{V}$ is constant).

Peper et al. (1994) argued that $V^{*}$ could be specified continuously by an $X(t) / \tau_{\text {tgt }}$ signal derived from perceptual estimates of $X(t)$ and $\tau_{\text {tgt. }}$. The descending motor command in this scheme is thus a specification of the required velocity $\left(V^{*}\right)$ for the intercepting effector. Thus, the requirement that $V=V^{*}$ is not achieved by explicitly comparing a perceptual estimate of $V$ with a perceptual estimate of $V^{*}$ (as in Equation 4). Rather, some mechanism ensures that the end-effector follows the speed trajectory specified by the command. Peper et al. noted that because $X(t) / \tau_{\text {tgt }}$ does not have the form of end-effector movement data recorded in experiments, the command signal cannot simply be directly proportional to $X(t) / \tau_{\text {tgt }}$. They proposed that the command is given by $\Omega(t) X(t) / \tau_{\text {tgt }}$, where $\Omega(t)$ is an activation function, $\Omega(t)$. However, choice of $\Omega(t)$ is post hoc: It is chosen so that the command signal has the same form as experimentally observed velocity profiles; it does not predict these profiles. A more refined and principled version of Peper et al.'s scheme has recently been developed by Dessing et al. (2002), using the VITE model of Bullock and Grossberg (1988), and will be discussed further below.

Tresilian's (1994b) formulation differed from Peper et al.'s (1994) in using an estimate of the interception location and being based on the equilibrium point hypothesis for motor control (see Feldman \& Levin, 1995; Latash, 1993). The formulation was based on simple, monotonic shifts of a hypothetical central command, known as the reciprocal ( $r$ ) command (Feldman \& Levin, 1995), that is sent to the spinal circuits associated with an agonist-antagonist pair of muscle groups. Together with the external load conditions, the level of the $r$ command determines the equilibrium position of a joint-level degree of freedom (e.g., flexion extension) or the equilibrium configuration of a multijoint system (Feldman \& Levin, 1995; Gribble, Ostry, Sanguineti, \& Laboissierre, 1998). In the single-joint case, movement from one joint position to another is generated by a shift in the $r$ command from a level $\left(r_{\mathrm{i}}\right)$ associated with the initial joint angle $\left(\varphi_{\mathrm{i}}\right)$ to a final level $\left(r_{\mathrm{d}}\right)$ associated with a desired angle $\left(\varphi_{\mathrm{d}}\right)$. Figure 8A illustrates such an $r$ command shift, which can be described using three parameters: the magnitude of the 


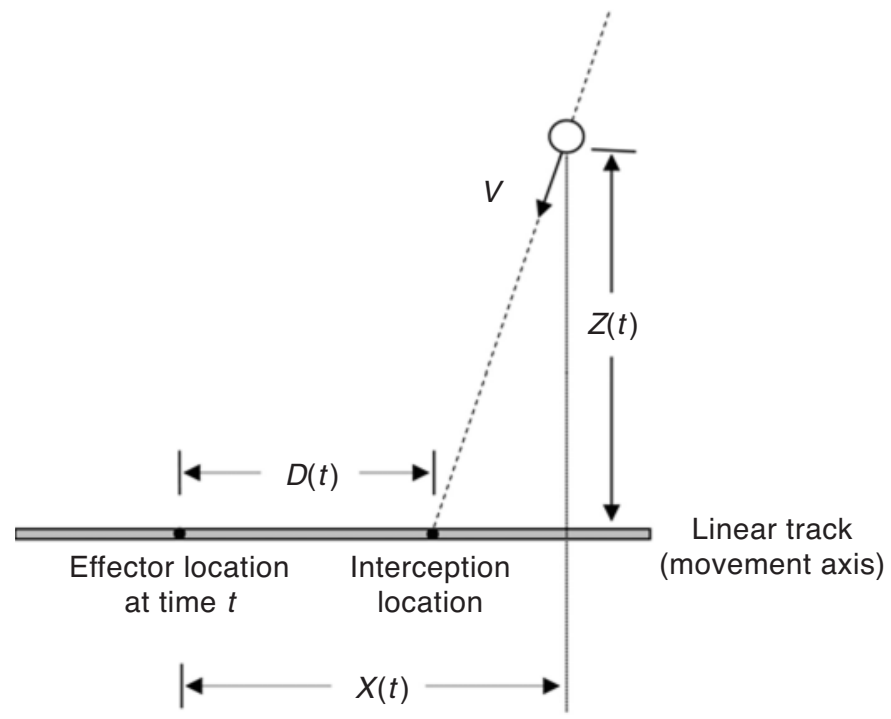

Figure 7. Approach configuration for interception assumed in Peper, Bootsma, Mestre, and Bakker's (1994) version of required velocity control. The interception location is where the target's path (dashed line) crosses the movement axis and is a distance $D(t)$ from the effector at time $t$.

shift $\left(r_{\mathrm{d}}-r_{\mathrm{i}}\right)$, the duration of the shift $(T)$, and its speed $(\omega)$. Figure $8 \mathrm{~B}$ illustrates the resulting movement from $\varphi_{\mathrm{i}}$ to $\varphi_{\mathrm{d}}$ : Movement duration $(M T)$ depends on $T$, movement amplitude depends on $\left(r_{\mathrm{d}}-r_{\mathrm{i}}\right)$, and movement speed depends on $\omega$. Thus, to achieve a certain $M T$, a value for $T$ is chosen that will result in the required $M T$ given a shift $\left(r_{\mathrm{d}}-r_{\mathrm{i}}\right) ; \omega$ is then given by $\left(r_{\mathrm{d}}-r_{\mathrm{i}}\right) / T$ (Latash, 1993).
To implement continuous control, the value of $\omega$ is continuously determined by the difference between the current value of the $r$ command, $r(t)$, and the required final level $\left(r_{\mathrm{d}}\right)$, divided by $\tau_{\text {tgt }}(t)$ :

$$
\omega(t)=\left[r_{\mathrm{d}}-r(t)\right] / \tau_{\mathrm{tgt}}(t)
$$

(Tresilian, 1994b, p. 362). This expression states that the $r$ command shifts at a rate such that the amount of shift
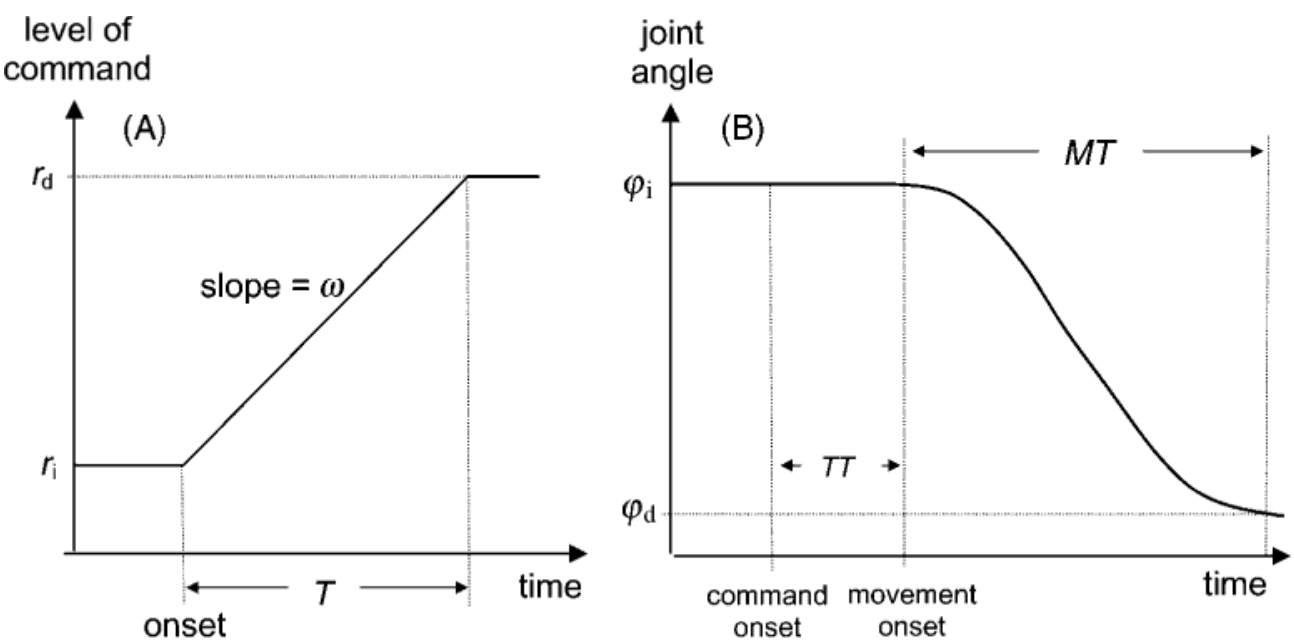

Figure 8. (A) Hypothetical shift in the central $r$ command underlying a single-joint positioning movement. The initial level of the command $\left(r_{\mathrm{i}}\right)$ defines the initial equilibrium position of the limb segment, and the final level $\left(r_{\mathrm{d}}\right)$ determines the final position. The shift from $r_{\mathrm{i}}$ to $r_{\mathrm{d}}$ takes a time $T$ and has a constant rate $\omega$. (B) The joint position as a function of time for the movement that results from the command in panel $A$. The initial position $\left(\varphi_{\mathrm{i}}\right)$ is the equilibrium angle associated with the initial command level $\left(r_{\mathrm{i}}\right)$; the final position $\left(\varphi_{\mathrm{d}}\right)$ is the equilibrium angle associated with the final level of the command $\left(r_{\mathrm{d}}\right)$. The time delay between the onset of the command and onset of movement is the transmission time (TT). 
remaining $\left[r_{\mathrm{d}}-r(t)\right]$, is completed in a time equal to $\tau_{\text {tgt }}(t)$. Equation 6 is a first-order differential equation in $r(\omega=d r / d t)$, and its solution is the $r$ command trajectory sent to the segmental neural circuitry. Thus, Equation 6 can be considered as a description of the neural dynamics of a network for generating the command signal, and in this respect, it is similar to the model proposed by Dessing et al. (2002).

\section{Movement Initiation in Continuous Control Schemes}

The timing of movement onset is not as critical to successful interception in continuous control schemes as it is in operational timing. Variation in onset time can be accommodated by the continuous control process that drives the temporal evolution of the movement. Some means of initiating the movement is still needed, of course, but since onset does not need to be precisely timed, TTC information may not be necessary; some covariant, such as the $R O E$, could be used instead. If a critical value of the $R O E$ were used for initiating the control process, $M T$ s would be longer to larger and slower targets, but as was described earlier, the value of $R O E_{\text {crit }}$ would have to vary with the distance to be moved, the size of the intercepting effector, and the viewing time. Such a scheme (a continuous control process initiated by critical values of the $R O E$ ) could certainly be made to fit the available data on hitting tasks (cf. Michaels et al., 2001).

\section{Separability of Time and Position Control in Continuous Models}

Tau-coupling in the formulation given above is separable: TTC information $\left(\tau_{\text {tgt }}\right)$ is explicitly represented, and a perceptual estimate of the interception location is used to direct the effector to the place of contact. Note that the latter is also required to define both $\tau_{\text {eff }}$ and $\tau_{\text {tgt }}$. In this interpretation, the tau-coupling control law (Equation 3 ) determines how the effector moves (its speed and acceleration) to an interception point determined by another process (not described).

RVC models are also separable, since they involve distinct representations of spatial (e.g., $X, D)$ and temporal $\left(\tau_{\text {tgt }}\right)$ quantities. For example, in Equation 6, the perceptual estimated interception location $\left(\varphi_{\mathrm{d}}\right)$ determines the final level of the $r$ command $\left(r_{\mathrm{d}}\right)$ and determines where the limb goes $\left(\varphi_{\mathrm{d}}\right)$. Another process (described by Equation 6) determines how the $r$ command evolves from its initial level $\left(r_{\mathrm{i}}\right)$ to the final (desired) level $\left(r_{\mathrm{d}}\right)$. If adjustments to the estimate of interception location take place during movement (i.e., $r_{\mathrm{d}}$ can change on line), time and position control are still separable in the sense just described (although they clearly interact). This is also the case in Peper et al.'s (1994) version of RVC, where the spatial information about $X$ (Figure 7) is used together with temporal information $\left(\tau_{\text {tgt }}\right)$ to determine the required speed $\left(V^{*}\right)$.

\section{Empirical Evidence for Continuous Control Strategies}

Data showing that unexpected disturbances elicit corrective responses during movement execution do not support continuous control over discrete control schemes. Perhaps because of the ambiguity concerning the interpretation of responses to perturbations, predictions derived from continuous control strategies concerning the temporal evolution of hypothesized controlled variables are sometimes derived and tested empirically. D. N. Lee, for example, has used this strategy to test continuous control hypotheses for hitting movements (D. N. Lee, Georgopoulos, Clark, Craig, \& Port, 2001), and others have used it for catching (McBeath et al., 1995; McLeod \& Dienes, 1996).

In D. N. Lee's tau-coupling hypothesis, the controller is attempting to make $\tau_{\text {eff }}$ equal to $\beta \tau_{\text {tgt }}$. Thus, the hypothesis predicts that during successful performance, a plot of $\tau_{\text {eff }}$ against $\tau_{\text {tgt }}$ should be a straight line of slope $\beta$ that passes through zero. Confirmation of this prediction has been interpreted as support for the tau-coupling theory (Lee et al., 2001). A related test of continuous control strategies is to show that kinematic performance variables, such as the position (Lee et al., 1983) or the velocity (Peper et al., 1994) of an effector, are functions of the hypothetical continuous control variable, and not of some other possible control variable. This strategy raises problems, because it does not provide evidence in favor of continuous control unless competing control strategies can be ruled out. It is notable that this was not done in those reports claiming support for continuous control. Subsequent analyses of these data sets have shown them to be consistent with preprogrammed control (Brouwer, Brenner, \& Smeets, 2003; Tresilian, 1994b, 1995). Tresilian (1994b) demonstrated that the results reported by Lee et al. (1983) and interpreted as evidence for continuous control were equally compatible with preprogrammed control. Brouwer, Brenner, and Smeets (2003) demonstrated that the results claimed in support of tau-coupling (e.g., Lee et al., 2001) are consistent with smooth interceptive movements involving no taucoupling. Indeed, such smooth movements can be generated by operational timing schemes. To provide support for continuous control, it is not sufficient to demonstrate that results are consistent with continuous control; it is necessary to show that the results are incompatible with alternative modes of control. This has been argued in detail previously (Brouwer, Brenner, \& Smeets, 2003; Tresilian, 1995).

\section{Empirical Evidence for RVC}

Montagne et al. (1999) derived predictions from the RVC hypothesis and tested them in an experiment, using a simple catching task. The major prediction concerned what Montagne et al. (1999) referred to as movement reversals - a reversal $\left(180^{\circ}\right.$ change $)$ in the direction of mo- 
tion of the intercepting effector. Montagne et al. (1999) observed that RVC predicts that such movement reversals would occur if the intercepting effector is initially stationary in the path of a moving target approaching along a straight path but at an angle to the observer, as shown in Figure 9. These reversals are expected to occur because $X(t)$, not $D(t)$, is used to provide a continuous estimate of the required velocity $\left(V^{*}\right)$. For example, if the intercepting effector (hand) is initially at $\mathrm{O}$ (Figure 9) and the target approaches $\mathrm{O}$ at an angle from the left (dashed path, Figure 9), $X(t)$ will initially be nonzero and will result in movement to the left. As the target approaches $\mathrm{O}$, the hand moves toward $\mathrm{O}$ to intercept it and so must reverse its direction of motion. If the target approaches along a perpendicular path (solid), reversals are not expected. Montagne et al. (1999) also observed that RVC predicts differences in the kinematics of movements toward the interception location (e.g., from Points $\mathrm{B}$ and $\mathrm{C}$ in Figure 9) when the target approaches the interception location from different directions due to differences in $X(t)$ [the hand should move faster for larger values of $X(t)]$.

The predictions were confirmed to some degree: Reversals were frequently observed (on about $56 \%$ of the trials) when the hand was initially at $\mathrm{O}$ and the target approached at an angle $\left( \pm 4^{\circ}\right)$, and the movement kinematics were different for different target approach angles and different initial hand positions $(\mathrm{O}, \mathrm{B}$, and $\mathrm{C}$, Figure 9). However, movement reversals were also observed (37\% of the trials) for perpendicular approaches $\left(0^{\circ}\right)$, and whether the kinematic variations were quantitatively consistent with the RVC model was not evaluated. In a recent modeling study, Dessing et al. (2002) showed that the versions of RVC formulated by Peper et al. (1994)

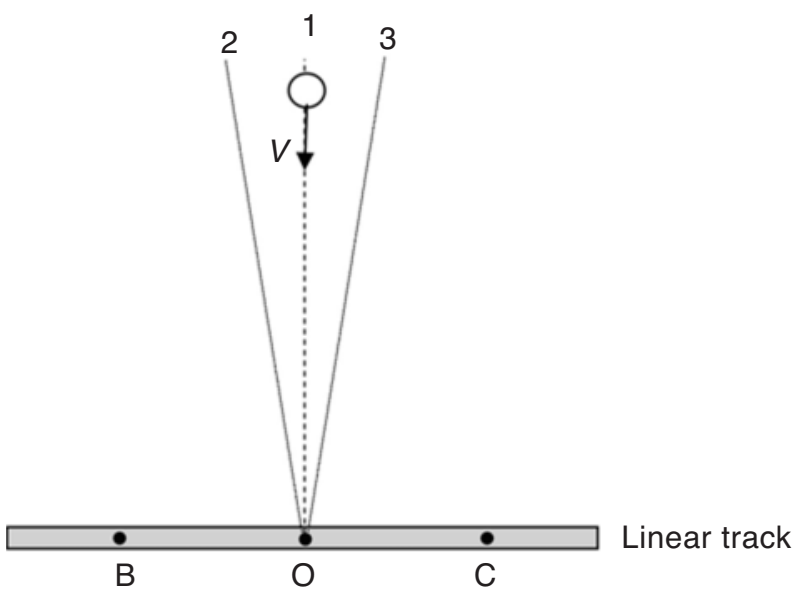

Figure 9. Schematic of the catching task used by Montagne, Laurent, Durey, and Bootsma (1999). A moving target (circle) moves at speed $V$ toward Point $O$ located on a straight linear track along which the participant's hand can move. The target moves along Path 1 perpendicular to the linear track $\left(0^{\circ}\right.$ approach, shown dashed) or along Paths 2 and 3 at a small angle $\left( \pm 4^{\circ}\right)$ to the perpendicular (dotted). The participant's hand can start either at $\mathrm{O}$ or at a point to the left or right (B or $\mathrm{C}$ ). and Bootsma et al. (1997) have difficulties accounting for the details of Montagne et al.'s (1999) data. They developed a version of the RVC hypothesis in Bullock and Grossberg's (1988) VITE model framework, which gave a much better account of the detailed pattern of results.

Overall, the results of Montagne et al.'s (1999) experiment are supportive of the use of on-line perceptual control: The presence of movement reversals strongly suggests that the movements are being regulated by perceptual information during performance. Montagne et al. (1999) argued that movement reversals can be explained only if $X(t)$ is the basis for control and that their data refute a strategy based on estimating the location of the interception point (e.g., one based on $D[t]$; Figure 7). Their version does not, however, provide an obvious explanation for why reversals should have been observed so often when the target approached along a perpendicular path or why they were sometimes not observed when the hand was at $\mathrm{O}$ and the target approached obliquely. If $D(t)$ is being estimated, movement reversals can occur if it is assumed that early estimates of $D(t)$ are error prone and corrupted by noise; in this case, initial estimates may be inaccurate, and the hand will move in the direction of the misestimate. Montagne et al.'s (1999) data appear insufficiently clear cut to decide between RVC based on $X(t)$ or RVC based on $D(t)$. The reason for basing RVC on $X(t)$ rather than on $D(t)$ is obscure. There is evidence to suggest that people are able to estimate where moving objects are going - their future spatial locations (Bootsma \& Peper, 1992; Regan \& Kaushal, 1994; Saxberg, 1987) whereas there is no evidence to suggest that people are able to make continuous estimates of $X(t)$, as is required by Peper et al.'s (1994) version of RVC. Of course, there are no data to suggest otherwise, either. However, given the currently available data, a version of RVC based on either continuous or discrete estimates of $D$ has the advantage of being consistent with known human capabilities.

Regardless of the version of RVC that is preferred, Montagne et al.'s (1999) results do not provide conclusive evidence for continuous control; the results are also consistent with discrete use of perceptual information. A movement reversal requires only a single correction: The person could make an initial movement to an erroneously perceived interception location but subsequently correct with a reversal.

\section{A Problem for Continuous Control}

Given that there is a delay between a visual stimulus and a corrective adjustment made in response to it, the effectiveness of error-corrective processes is greater for movements of longer duration. Greater accuracy and precision demands and/or greater uncertainty would thus be expected to elicit movements with longer $M T$ s and lower speeds, if on-line control were being used. This is the usual explanation advanced for the Fitts'-law-type speed-accuracy tradeoff in movements aimed at stationary targets (Meyer et al., 1988; Schmidt, 1988). However, the reverse pattern is observed in rapid interceptive actions, and briefer, faster movements are found to be 
more temporally precise (Newell, 1980; Tresilian et al., 2003). These observations find a ready explanation in the operational timing framework, as was described earlier. In contrast, on-line control offers no clear rationale for why demands for greater temporal accuracy should elicit responses that offer less opportunity to use it.

This is not to say that on-line control models, such as $\mathrm{RVC}$, could not be modified to produce the response patterns so far described for hitting movements, but only that such models offer no clear rationale for these patterns. Nevertheless, these models would appear to have some difficulty accounting for the results reported by Tresilian et al. (2003) concerning the responses of participants in a coincidence anticipation task. This task required participants to briefly press down on a force transducer with the index finger at the same moment that a moving target reached a designated location. The force pulses produced were very brief $(<150 \mathrm{msec})$ and showed a pattern similar to that of hitting movements: The pulses reached greater peak forces and were of shorter duration when the target moved faster or was smaller in size. The pulses were presumably visually open loop and consistent with preprogrammed control of timed responses to moving targets.

\section{DISCUSSION}

The analysis presented in previous sections shows that neither preprogrammed nor continuous control provides a completely satisfactory account of how interceptions are timed. For rapid hitting and coincidence anticipation tasks, the preprogrammed account is more plausible and is compatible with the dominant view that aimed movements involve an initial preprogrammed component (e.g., Desmurget \& Grafton, 2000; Elliott et al., 2001). Interceptive aiming may also involve an initial preprogrammed component, with exteroceptive feedback control playing a role only after a time delay. For movement involving elbow and shoulder flexion-extensions, data suggest that the delay between a visual stimulus event and an observable response in an ongoing movement lies in the range of 120-200 msec (Paillard, 1996; Saunders \& Knill, 2003). If the preprogrammed component is more important than on-line, exteroceptive control in rapid hitting actions, people might adopt a preprogrammed strategy, as will be discussed next.

\section{The Preprogrammed Strategy and Speed-Accuracy Tradeoffs in Hitting Tasks}

If exteroceptive feedback were useful, it would make sense to respond to increased demands for spatiotemporal accuracy and precision in a manner that would increase the opportunity to make use of it. It is widely accepted that longer $M T$ s for movements requiring greater spatial accuracy is the result of a strategy for using feedback (Elliott et al., 2001; Schmidt, 1988). If exteroceptive feedback is not useful, a different strategy for improving spatiotemporal accuracy is needed. As was argued earlier, making briefer, faster movements to targets requir- ing greater temporal accuracy represents such a strategy: It reduces the opportunity to use feedback but improves temporal accuracy. Use of a preprogrammed strategy in interceptive hitting is supported by the results of a recent study in which spatial (directional) accuracy requirements were manipulated while temporal accuracy requirements were kept constant (Tresilian, Plooy, \& Carroll, 2004). $M T$ was largely unaffected by changes in spatial accuracy demands (as determined by four target sizes: 2, 4, 8, and $16 \mathrm{~cm}$ ), but when reliable effects were observed, they were for briefer, more rapid movements to smaller targets. In accord with these findings, Howarth and Beggs (1985) reviewed data showing that the directional accuracy and precision of movements aimed at stationary targets under visually open-loop conditions is virtually unaffected by movement duration. Thus, in the absence of visual feedback, the spatial accuracy of hitting is unlikely to be improved by moving more slowly; hence, there is no advantage to be gained by doing so. Temporal accuracy, in contrast, is improved by moving more rapidly. Results reported by Schmidt and Sherwood (1982) showed an unexpected improvement in spatial precision for very fast, brief $(M T<100 \mathrm{msec})$, presumably ballistic movements, raising the possibility that in open-loop conditions, briefer, faster movements may sometimes increase both spatial and temporal precision (for further discussion, see Tresilian et al., 2004).

These findings all point to the possibility of a preprogrammed strategy for hitting moving targets as follows: Meet demands for greater accuracy and precision by altering performance in a way that improves the effectiveness of the preprogrammed component of aimed movement but does not improve the effectiveness of feedback control.

It has been proposed that when a moving target is intercepted, there are two tradeoffs at work (Brouwer et al., 2000; Schmidt, 1988; Tresilian \& Lonergan, 2002): (1) a Fitts-type speed-accuracy tradeoff, in which $M T$ increases in response to greater spatial accuracy demands, allowing more use of feedback, and (2) a temporal tradeoff of the type detailed here, in which $M T$ decreases in response to greater temporal accuracy demands and target speed (Equation 1), permitting less use of feedback. The preprogrammed strategy is a manifestation of the temporal tradeoff and is expected in circumstances in which accurate and precise timing is critical and the temporal tradeoff dominates over the Fitts-type tradeoff. Hitting fast-moving targets may be a circumstance in which the temporal tradeoff dominates. Note that this strategy does not deny the existence of on-line, feedback control in these movements, but only that feedback plays little useful role in rapid hitting actions. The extent to which feedback plays a role in hitting is an empirical question, considered next.

\section{Is Exteroceptive Feedback Used in Rapid Hitting Tasks?}

Much of the data demonstrating on-line corrective adjustments to manual interceptive actions come from ex- 
periments in which the task did not involve hitting fastmoving targets (e.g., Brenner et al., 1998; Brouwer, Middelburg, Smeets, \& Brenner, 2003; D. Lee et al., 1997; Montagne et al., 1999). For example, Montagne et al. (1999) used a catching task, rather than a hitting task, that was performed using slow (peak speeds $\approx 0.2 \mathrm{~m} / \mathrm{sec}$ ) movements of relatively long duration $(M T \mathrm{~s} \geq 500 \mathrm{msec}$, approximately) in which the hand decelerated on approach to contact. Similarly, the interception task of Port and colleagues (D. Lee et al., 1997; Port, D. Lee, Dassonville, \& Georgopoulos, 1997) involved slow-moving targets, the hand decelerating on approach to the interception location, and $M T \mathrm{~s}$ in the approximate range of $500-1,800 \mathrm{msec}$ (when corrective submovements were observed). In these circumstances, the Fitts-type strategy might be expected, since there is no need to move rapidly and there is time to use feedback.

Brenner, Smeets, and colleagues studied a rapidly executed hitting task and obtained data that they interpreted in terms of an on-line control model (Brenner et al., 1998; Brouwer, Middelburg, et al., 2003). In their task, a computer-generated target moved in a straight line over a plane surface, and the participant attempted to hit the target with a handheld rod (Figure 10). Movements in these experiments were brief (averaging between 200 and $400 \mathrm{msec}$ ), with peak speeds exceeding $2 \mathrm{~m} / \mathrm{sec}$. Recently, these authors have interpreted their results as indicating that timing is not adjusted on line (Brouwer et al., 2000), whereas movement direction is (Brouwer, Middleburg, et al., 2003). On-line adjustment of movement direction in this task may be facilitated by two factors. (1) Slow target speeds were used $(0.06 \leq$ target speed $<0.25 \mathrm{~m} / \mathrm{sec}$ ), as compared, for example, with speeds an order of magnitude greater $(1.0 \mathrm{~m} / \mathrm{sec} \leq$ target speed $<2.5 \mathrm{~m} / \mathrm{sec}$ ) in Tresilian et al. (2004) and two orders of magnitude greater in baseball, cricket, and tennis (Regan, 1992; Watts \& Bahill, 1990). (2) In Brenner and colleagues' task, the person is free to move in the direction of target motion, allowing approach to interception to be a combination of the pursuit and the perpendicular geometries (Figures 1E and 10). In our experiments, the participants were restricted to the perpendicular approach geometry (Figure 1D). Thus, a preprogrammed strategy for timing may be adopted in Brenner and colleagues' task, but there is some opportunity to use on-line feedback to fine-tune movement direction (see Brouwer et al., 2000).

The general applicability of the preprogrammed strategy in rapid hitting tasks is called into question by evidence suggesting that on-line control could be involved for some longer duration movements in our $1-d f$ hitting experiments (Tresilian \& Lonergan, 2002), particularly if the greater $M T$ was associated with a larger movement amplitude (D in Figure 3). Under these circumstances, a significant number of movements exhibited an initial slow phase, followed by a later rapid phase. Figure 11 shows some example velocity profiles of these biphasic movements. These were rare $(<2 \%$ of all the trials) or nonexistent for short-amplitude movements (short $M T \mathrm{~s}$ ) but became increasingly more common when the amplitude was greater (the $M T$ was longer). Movements with the greatest amplitude $(38.5 \mathrm{~cm})$ were biphasic on about $50 \%$ of the trials in the three experiments reported in Tresilian and Lonergan. These results suggest a discrete corrective adjustment to an ongoing movement; whether they can be accommodated by a purely preprogrammed control process will be considered next.

\section{A Biphasic Preprogrammed Model}

A biphasic movement could be produced by a biphasic, preprogrammed control strategy with two preestablished components that, once elicited, are both executed in visually open-loop mode. The first component moves

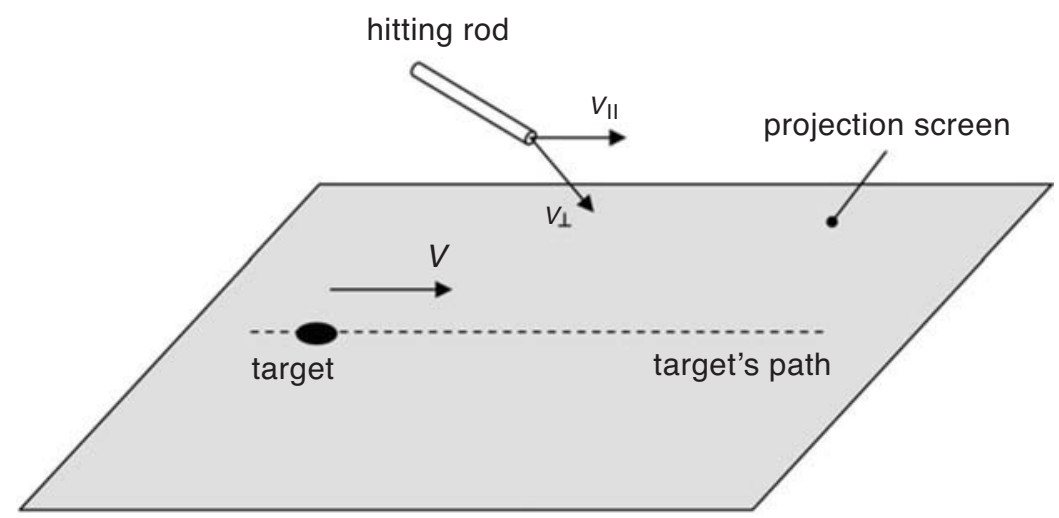

Figure 10. Schematic illustration of the hitting task used by Brenner, Smeets, and colleagues. The participant holds a hitting rod at an initial location displaced from a plane surface (projection screen). Back projected onto this screen is the image of a moving target (and a background texture over which it moves). The target moves at some constant speed $V$ along a straight path. The component of rod tip velocity perpendicular to the screen is labeled $v_{\perp}$; the component parallel to the screen in the direction of the target motion is labeled $v_{\|}$. 


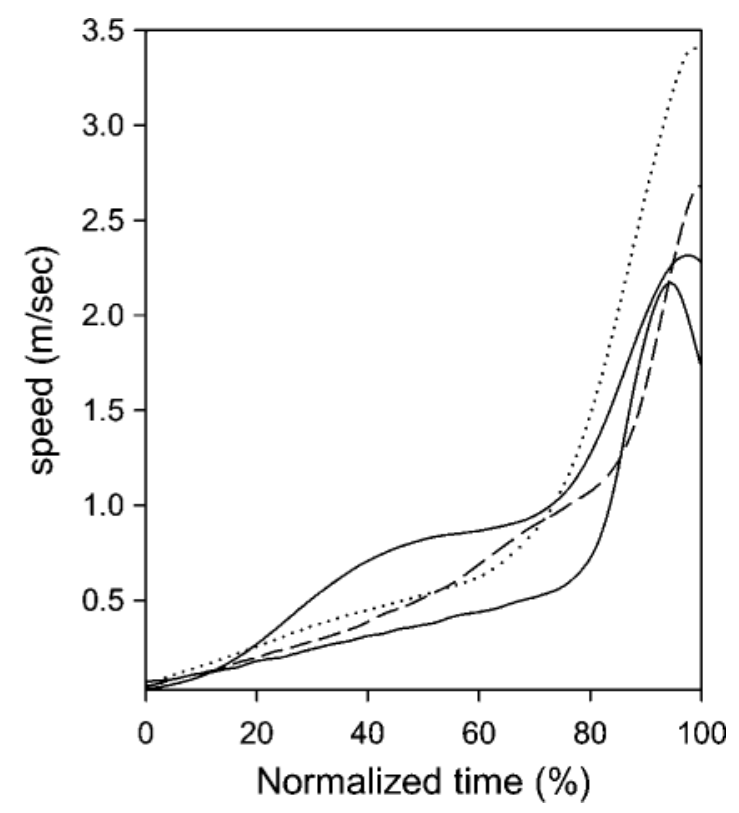

Figure 11. Four example velocity profiles (up to the moment of target contact) that exhibited an initial slow phase followed by a second more rapid phase. All have been time normalized to run from zero to $100 \%$ of total movement time. All these examples were taken from the participant who showed the most pronounced biphasic pattern of the 18 people who participated in the experiments reported by Tresilian and Lonergan (2002).

the effector toward the interception location relatively slowly, and at some point, a faster, second component is elicited. The second component is not a correction to the first but is preplanned and triggered by stimulus information at an appropriate time. This second component is brief and fast and so exploits the greater temporal precision associated with such movements.

A possible implementation of the biphasic scheme for hitting in the $1-d f$ task would involve initiating the first component at one critical value of $T T C_{\text {tgt }}\left(T T C_{\text {crit, } 1}\right)$ and the second component at a smaller value $\left(T T C_{\mathrm{crit}, 2}\right)$. The problem is then to ensure that the second component moves the effector over the remaining distance in a time equal to $T T C_{\text {crit }, 2}-(T T+P T)$ (see the Preprogrammed Control of Timing section). One hypothetical method for doing this in the $1-d f$ task (Figure 2 ) is to use the concepts described earlier in the derivation of Equation 6.

Consider first a monophasic preprogrammed hitting movement. Figure 12 shows the temporal evolution of a hypothetical preprogrammed central command signal for such a hitting movement (top graph). The resulting movement is represented schematically in the bottom graph, which shows a schematic of the position of the intercepting effector (distance from interception location, solid line) and its tangential speed as functions of time. The command $(R)$ is the multijoint version of the $r$ command described earlier (see, e.g., Feldman \& Levin, 1995). Each value of the command, if held fixed, would correspond to a specific equilibrium position of the intercepting effector. In the figure, $R_{\mathrm{i}}$ is the command level associated with the initial position of the effector, and $R_{\mathrm{D}}$ is the level associated with a position a distance $D$ from the initial position - the hitting location (Figure 3). Since the effector does not stop at the hitting location, $R_{\mathrm{D}}$ is not the final level of the command $\left(R_{\mathrm{f}}\right)$. The command shifts from $R_{\mathrm{i}}$ to $R_{\mathrm{D}}$ in a time $T$ at a constant rate, beginning a short time $(P T)$ after $T T C_{\text {tgt }}$ reaches the critical value. The time $T$ must be selected so as to produce the required $M T$. It appears reasonable to assume that there exists a one-to-one relationship between command shift duration $(T)$ and $M T$ (Latash, 1993; St-Onge, Adamovich, \& Feldman, 1997). This means that $M T=f(T)$, where $f$ is the function that maps command duration into movement duration and depends on the effector neuromechanics. Thus, if the desired $M T$ is $M T_{\text {prog }}$, the command duration $(T)$ would be given by $f^{-1}\left(M T_{\text {prog }}\right)$, where $f^{-1}$ is the inverse of $f$. In other words, the command duration $T$ is derived from the desired $M T$ using some kind of inverse model $\left(f^{-1}\right)$ of the neuromechanics (cf. Wolpert, Miall, \& Kawato, 1998).

Figure 13 illustrates the biphasic case that involves a first (slow) preprogrammed component initiated at one critical value of $T T C_{\text {tgt }}\left(T T C_{\text {crit }, 1}\right)$, followed by a second component initiated at a smaller $T T C_{\mathrm{tgt}}$ value $\left(T T C_{\mathrm{crit}, 2}\right)$. A time $P T$ after $T T C_{\text {tgt }}$ reaches $T T C_{\text {crit }, 1}$, the command for the first component begins. A short time later, $T T C_{\mathrm{tgt}}$

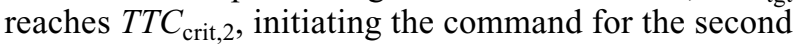
component after a time PT. At this moment, the initial command component has executed for a time $T 1$ and reached a level $R_{\mathrm{x}}$ leaving a shift of $R_{\mathrm{D}}-R_{\mathrm{X}}$ to reach the level associated with the hitting location $\left(R_{\mathrm{D}}\right)$. This remaining shift must be completed in a time that ensures that the remaining movement is completed in a time equal to $T T C_{\text {crit, } 2}-(T T+P T)$. According to the argument presented in the last paragraph, the shift duration should then be equal to $f^{-1}\left[T T C_{\mathrm{crit}, 2}-(T T+P T)\right]$, which is denoted $T 2$ in Figure 13.

The simple biphasic scheme of Figure 13 is developed here only to a degree sufficient to illustrate how a preprogrammed control strategy might work to produce the biphasic movement patterns observed in experiments. Distinguishing this model from alternatives, such as a discrete correction model, is difficult: Both models predict that later phases should be absent if vision of the target is prevented. However, recent work in which the auditory startle reflex has been used suggests a possible method (Carlsen, Hunt, Inglis, Sanderson, \& Chua, 2003; Valls-Sole, Rothwell, Goulart, Cossu, \& Munoz, 1999). This work has shown that prepared arm movement responses can be triggered by a startling auditory stimulus; Carlsen et al. showed that this is also true for the secondary component of a prepared two-component movement sequence. If the second phase of the biphasic hitting movements is preprogrammed, it may be possible to trigger it by using a startling stimulus, following the method of Carlsen et al. This would not be possible were the second phase of a biphasic movement a corrective 


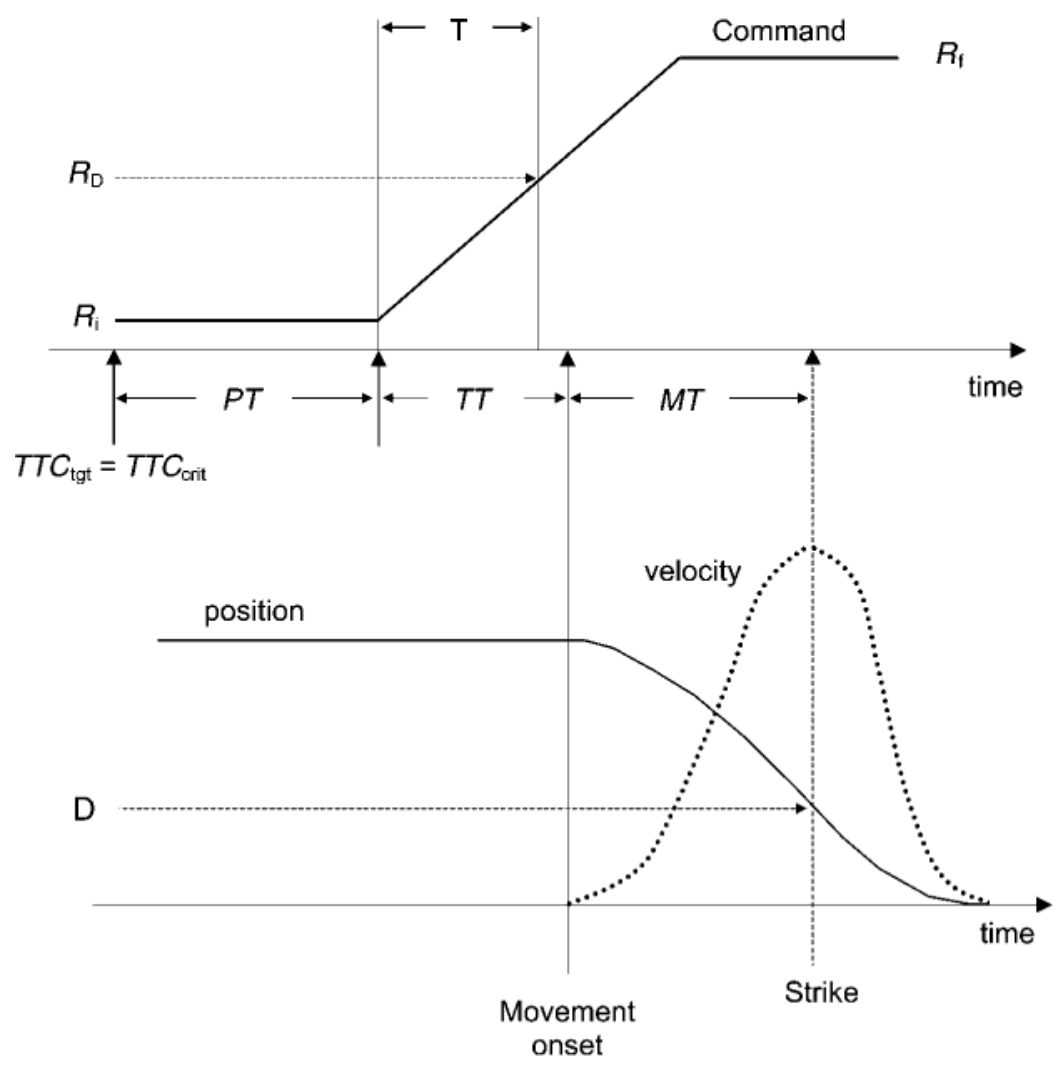

Figure 12. Illustration of a possible implementation of the single-phase preprogrammed control scheme described in the text, using the tools of the equilibrium point hypothesis (Latash, 1993). The command that drives the intercepting effector $(R$ command) has a ramp form (top, solid line) and leads to movement of the form illustrated by the position (solid) and velocity (dotted) profiles (bottom). PT, perceptual transmission time; $T T$, motor command transmission time.

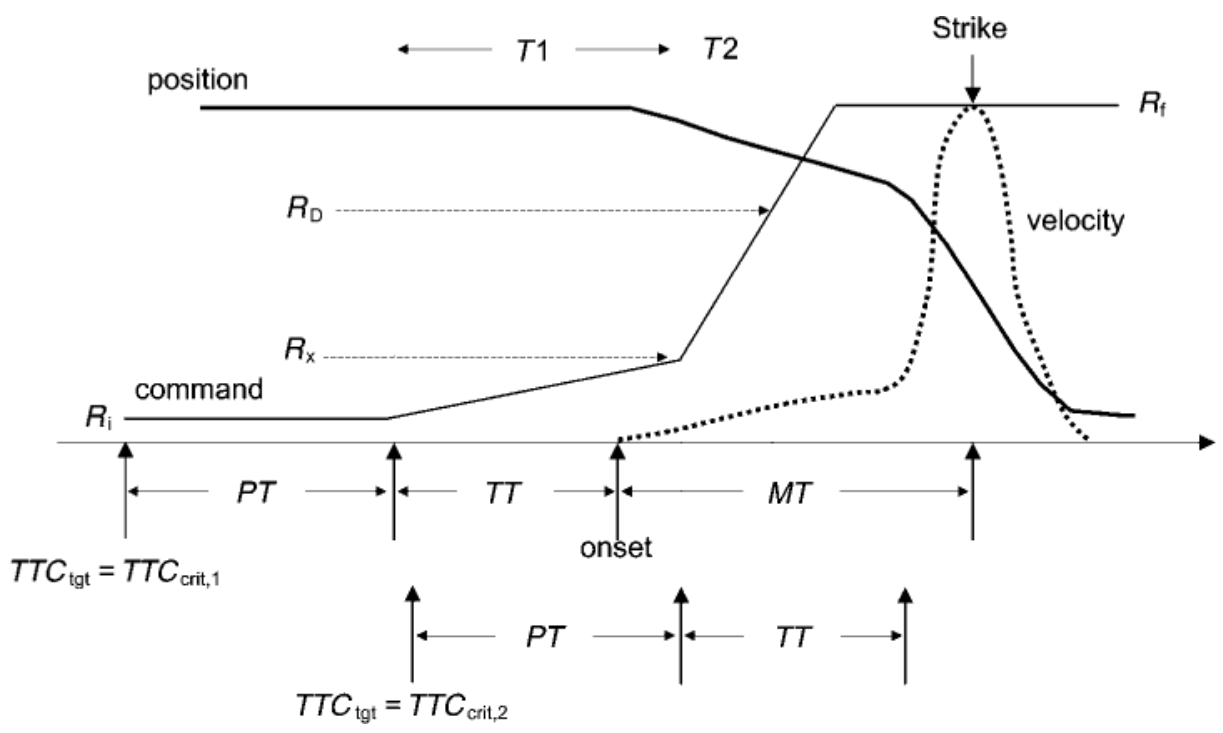

Figure 13. Illustration of a possible implementation of the biphasic preprogrammed control scheme described in the text. The format is similar to that of Figure 12 (see the text for more details). 
adjustment made on the basis of visual feedback. Thus, if a biphasic movement could be triggered by a startling stimulus or if such a stimulus could elicit the second component, this would provide strong support for the preprogrammed strategy.

\section{Conclusions}

A suitably formulated preprogrammed model is able to provide a plausible account of performance for some interceptive actions. These actions may form a class of interceptions in which the temporal tradeoff is dominant over the Fitts-type tradeoff, leading to what was referred to as the preprogrammed control strategy. This class includes manual hitting-type interceptions of faster moving targets $(» 20 \mathrm{~cm} / \mathrm{sec})$ that are executed rapidly $(M T<$ $500 \mathrm{msec}$ ). There is little or no evidence for feedback adjustment of movement timing in hitting movements of this type. What evidence there is can be accommodated by the biphasic preprogrammed model just described or attributed to adjustment to movement direction. Thus, movement timing in rapid hitting actions may be executed in a completely preprogrammed mode. Other types of manual interception - notably, catches that involve the pursuit and receding configurations shown in Figure 1are likely to involve on-line control. Whether this is of the continuous type proposed by the tau-coupling and RVC models remains to be determined.

\section{REFERENCES}

Abernethy, B. (1990). Expertise, visual search and information pickup in squash. Perception, 19, 63-77.

Alexander, R. M. (1997). A minimum energy cost hypothesis for human arm trajectories. Biological Cybernetics, 76, 97-105.

BAIRSTOW, P. J. (1987). Analysis of hand movement to moving targets. Human Movement Science, 6, 205-231.

Ball, C. T., \& Glencross, D. (1985). Developmental differences in a coincident timing task under speed and time constraints. Human Movement Science, 4, 1-15.

Bootsma, R. J., FaYt, V., ZaAL, F. T. J. M., \& Laurent, M. (1997). On the information-based regulation of movement: Things Wann (1996) might want to consider. Journal of Experimental Psychology: Human Perception \& Performance, 23, 1282-1289.

Bootsma, R. J., \& Peper, C. E. (1992). Predictive visual information sources for the regulation of action with special emphasis on catching and hitting. In L. Proteau \& D. Elliott (Eds.), Vision and motor control (pp. 285-314). Amsterdam: Elsevier.

Bootsma, R. J., \& VAN Wieringen, P. C. W. (1990). Timing an attacking forehand drive in table tennis. Journal of Experimental Psychology: Human Perception \& Performance, 16, 21-29.

BREEN, J. L. (1967). What makes a good hitter? Journal of Health, Physical Education, \& Recreation, 38, 36-39.

Brenner, E., Smeets, J. B. J., \& De Lussanet, M. (1998). Hitting moving targets: Continuous control of the acceleration of the hand on the basis of the target's velocity. Experimental Brain Research, 122, 467-474.

Brogan, W. L. (1990). Modern control theory. New York: Prentice-Hall.

Brouwer, A.-M., Brenner, E., \& Smeets, J. B. J. (2000). Hitting moving objects: The dependency of hand velocity on the speed of the target. Experimental Brain Research, 133, 242-248.

Brouwer, A.-M., Brenner, E., \& SMeETS, J. B. J. (2003). When is behavioral data evidence for a control theory? Tau-coupling revisited. Motor Control, 7, 103-110.

Brouwer, A.-M., Middelburg, T., Smeets, J. B. J., \& Brenner, E.
(2003). Hitting moving targets: A dissociation between the use of the target's speed and direction of motion. Experimental Brain Research, 152, 368-375.

Bullock, D., \& Grossberg, S. (1988). Neural dynamics of planned arm movements: Emergent invariants and speed-accuracy properties during trajectory formation. Psychological Review, 95, 49-90.

Caljouw, S. R., van der Kamp, J., \& Savelsberg, G. J. P. (2004). Timing of goal-directed hitting: Impact requirements change the informationmovement coupling. Experimental Brain Research, 155, 135-144.

Carlsen, A. N., Hunt, M. A., Inglis, T. J., Sanderson, D. J., \& ChuA, R. (2003). Altered triggering of a prepared movement by a startling stimulus. Journal of Neurophysiology, 89, 1857-1863.

Carpenter, R. H. S. (1977). Movements of the eyes. London: Pion.

Chardenon, A., Montagne, G., Buekers, M. J., \& Laurent, M. (2002). The visual control of ball interception during human locomotion. Neuroscience Letters, 334, 13-16.

Cutting, J. E., Vishton, P. M., \& Braren, P. A. (1995). How we avoid collisions with stationary and moving obstacles. Psychological Review, 102, 627-651.

DeLucia, P. R. (1991). Pictorial and motion-based information for depth perception. Journal of Experimental Psychology: Human Perception \& Performance, 17, 738-748.

DeLucia, P. R., \& WARREN, R. (1994). Pictorial and motion-based depth information during active control of self-motion: Size-arrival effects on collision avoidance. Journal of Experimental Psychology: Human Perception \& Performance, 20, 783-798.

De Lussanet, M. H. E. (2001). The control of interceptive arm movements. Rotterdam: Erasmus University Press.

De Lussanet, M. H. E., Smeets, J. B. J., \& Brenner, E. (2001). The effect of expectations on hitting moving targets: Influence of the preceding target's speed. Experimental Brain Research, 137, 246-248.

Desmurget, M., \& Grafton, S. (2000). Forward modeling allows feedback control for fast reaching movements. Trends in Cognitive Sciences, 4, 423-431.

Dessing, J. C., Bullock, D., Peper, C. L., \& Beek, P. J. (2002). Prospective control of manual interceptive actions: Comparative simulations of extant and new model constructs. Neural Networks, 15, 163-179.

Elliott, D., Helsen, W. F., \& ChUA, R. (2001). A century later: Woodworth's (1899) two component model of goal-directed aiming. Psychological Bulletin, 127, 342-357.

Elliott, D., Zuberec, S., \& Milgram, P. (1994). The effects of periodic visual occlusion on ball catching. Journal of Motor Behavior, 26, 113-122.

ENOKA, R. (2002). Neuromechanics. Champaign, IL: Human Kinetics.

Feldman, A. G., \& Levin, M. F. (1995). The origin and use of positional frames of reference in motor control. Behavioral \& Brain Sciences, 18, 723-744.

FITCH, H., \& TuRvey, M. T. (1978). On the control of activity: Some remarks from an ecological point of view. In D. Landers \& R. Christina (Eds.), Psychology of motor behavior and sport (pp. 3-35). Champaign, IL: Human Kinetics.

Fleury, M., Basset, F., Bard, C., \& Teasdale, N. (1998). Target speed alone influences the latency and temporal accuracy of interceptive action. Canadian Journal of Experimental Psychology, 52, 84-92.

GRAY, R. (2002a). Behavior of college baseball players in a virtual batting task. Journal of Experimental Psychology: Human Perception \& Performance, 28, 1131-1148.

Gray, R. (2002b). "Markov at the bat": A model of cognitive processing in baseball batters. Psychological Science, 13, 543-548.

Gray, R., \& Regan, D. (1998). Accuracy of estimating time to collision using binocular and monocular information. Vision Research, 38, 499-512.

Green, D. M., \& Swets, J. A. (1966). Signal detection theory and psychophysics. New York: Wiley.

Gribble, P. L., Ostry, D. J., Sanguineti, V., \& Laboissierre, R. (1998). Are complex control signals required for human arm movement? Journal of Neurophysiology, 79, 1409-1424.

Harris, C. M., \& Wolpert, D. M. (1998). Signal dependent noise determines motor planning. Nature, 384, 780-784. 
Howarth, C. I., \& Beggs, W. D. A. (1985). The control of simple movements by multisensory information. In H. Heuer, U. Kleinbeck, \& K.-H. Schmidt (Eds.), Motor behavior: Programming, control, and acquisition (pp. 125-151). Berlin: Springer-Verlag.

Hubbard, A. W., \& SenG, C. N. (1954). Visual movements of batters. Research Quarterly, 25, 42-57.

JaGaCinski, R. J., RepPerger, D. W., Ward, S. L., \& Moran, M. S. (1980). A test of Fitts' law with moving targets. Human Factors, 22, 225-233.

KAWATO, M. (1999). Internal models for motor control and trajectory planning. Current Opinion in Neurobiology, 9, 718-727.

Latash, M. L. (1993). Control of human movement. Chicago: Human Kinetics.

Laurent, M., Montagne, G., \& Savelsbergh, G. J. P. (1994). The control and coordination of one-handed catching: The effect of temporal constraints. Experimental Brain Research, 101, 314-322.

Lee, D., Port, N. L., \& Georgopoulos, A. P. (1997). Manual interception of moving targets: II. On-line control of overlapping submovements. Experimental Brain Research, 116, 421-433.

LEE, D. N. (1980). Visuo-motor coordination in space-time. In G. E. Stelmach \& J. Requin (Eds.), Tutorials in motor behavior (pp. 281295). Amsterdam: North-Holland.

LEE, D. N. (1998). Guiding movement by coupling taus. Ecological Psychology, 10,221-250.

Lee, D. N., Georgopoulos, A. P., Clark, M. J., Craig, C. M., \& Port, N. L. (2001). Guiding contact by coupling the taus of gaps. Experimental Brain Research, 139, 151-159.

LeE, D. N., Young, D. S., Reddish, P. E., Lough, S., \& ClayTON, T. M. H. (1983). Visual timing in hitting an accelerating ball. Quarterly Journal of Experimental Psychology, 35A, 333-346.

Mason, A. H., \& Carnahan, H. (1999). Target viewing time and velocity effects on prehension. Experimental Brain Research, 127, 8394.

McBeath, M. K., Shaffer, D. M., \& Kaiser, M. K. (1995). How baseball outfielders determine where to run to catch fly balls. Science, 268, 569-573.

MCLEOD, P., \& Dienes, Z. (1996). Do fielders know where to go to catch the ball or only how to get there? Journal of Experimental Psychology: Human Perception \& Performance, 22, 531-543.

MEYer, D. E., Kornblum, S., Abrams, R. A., WRIGHT, C. E., \& SMith, J. E. K. (1988). Optimality in human motor performance: Ideal control of rapid aimed movement. Psychological Review, 95, 340-370.

Michaels, C. F., \& Oudejans, R. R. D. (1992). The optics and actions of catching fly balls: Zeroing out optic acceleration. Ecological Psychology, 4,199-222.

Michaels, C. F., Zeinstra, E. B., \& Oudejans, R. R. D. (2001). Information and action in punching a falling ball. Quarterly Journal of Experimental Psychology, 54A, 69-93.

Montagne, G., Fraisse, F., Ripoll, H., \& Laurent, M. (2000). Perception-action coupling in an interceptive task: First-order timeto-contact as an input variable. Human Movement Science, 19, 5972 .

Montagne, G., Laurent, M., Durey, A., \& Bootsma, R. (1999). Movement reversals in ball catching. Experimental Brain Research, 129, 87-92.

NEWELL, K. M. (1980). The speed-accuracy paradox in movement control: Errors of time and space. In G. E. Stelmach \& J. Requin (Eds.), Tutorials in motor behavior (pp. 501-510). Amsterdam: North-Holland.

Newell, K. M., Hoshizaki, L. E. F., Carlton, M. J., \& Halbert, J. A. (1979). Movement time and velocity as determinants of movement timing accuracy. Journal of Motor Behavior, 11, 49-58.

Paillard, J. (1996). Fast and slow feedback loops for the visual correction of spatial errors in a pointing task: A reappraisal. Canadian Journal of Physiology \& Pharmacology, 74, 401-417.

PePer, C. L., Bootsma, R. J., Mestre, D., \& BaKker, F. (1994). Catching balls: How to get the hand to the right place at the right time. Journal of Experimental Psychology: Human Perception \& Performance, 20,591-612.

PorT, N. L., LeE, D., Dassonville, P., \& Georgopoulos, A. P. (1997).
Manual interception of moving targets: I. Performance and movement initiation. Experimental Brain Research, 116, 406-420.

Poulton, E. C. (1950). Perceptual anticipation and reaction time. Quarterly Journal of Experimental Psychology, 2, 99-112.

Poulton, E. C. (1957). On prediction in skilled movements. Psychological Bulletin, 54, 467-478.

REGAN, D. (1992). Visual judgements and misjudgements in cricket, and the art of flight. Perception, 21, 91-115.

Regan, D., \& Hamstra, S. J. (1993). Dissociation of discrimination thresholds for time to contact and for rate of angular expansion. $\mathrm{Vi}$ sion Research, 33, 447-462.

Regan, D., \& KAUSHAL, S. (1994). Monocular discrimination of the direction of motion in depth. Vision Research, 34, 163-177.

Saltzman, E., \& Kelso, J. A. S. (1987). Skilled actions: A task-dynamic approach. Psychological Review, 94, 84-106.

SAUNDERS, J. A., \& KNILL, D. C. (2003). Humans use continuous visual feedback from the hand to control fast reaching movements. Experimental Brain Research, 152, 341-352.

SAXBERG, B. V. H. (1987). Projected free fall trajectories: 2. Human experiments. Biological Cybernetics, 56, 177-184.

SchmidT, R. A. (1969). Movement time as a determiner of timing accuracy. Journal of Experimental Psychology, 79, 43-47.

SCHMidT, R. A. (1988). Motor control and learning: A behavioral emphasis. Champaign, IL: Human Kinetics.

SCHMIDT, R. A., \& SHERwood, D. E. (1982). An inverted-U relation between spatial error and force requirements in rapid limb movements: Further evidence for the impulse variability model. Journal of Experimental Psychology: Human Perception \& Performance, 8, 158170.

Schmidt, R. A., Zelaznik, H. [N.], Hawkins, B., Frank, J. S., \& QUINN, J. T., JR. (1979). Motor-output variability: A theory for the accuracy of rapid motor acts. Psychological Review, 47, 415-451.

Senot, P., Prevost, P., \& McIntyre, J. (2003). Estimating time to contact and impact velocity when catching an accelerating object with the hand. Journal of Experimental Psychology: Human Perception \& Performance, 29, 219-237.

SHARP, R. H., \& Whiting, H. T. A. (1974). Exposure and occluded duration effects in a ball catching skill. Journal of Motor Behavior, 3, 139-147.

Sharp, R. H., \& Whiting, H. T. A. (1975). Information-processing and eye-movement behavior in a ball catching skill. Journal of Human Movement Studies, 1, 124-131.

Sidaway, B., Fairweather, M., Sekiya, H., \& McNitt-Gray, J. (1996). Time-to-collision estimation in a simulated driving task. Human Factors, 38, 101-113.

Smith, M. R. H., Flach, J. M., Dittman, S., \& Stanard, T. (2001). Monocular optical constraints on collision control. Journal of Experimental Psychology: Human Perception \& Performance, 27, 395-410.

SpARrow, W. A. (Ed.) (2000). Energetics of human activity. Champaign, IL: Human Kinetics.

St-Onge, N., Adamovich, S. V., \& Feldman, A. G. (1997). Control processes underlying elbow flexion movements may be independent of kinematic and electromyographic patterns: Experimental study and modelling. Neuroscience, 79, 295-316.

Tresilian, J. R. (1994a). Approximate information sources and perceptual variables in interceptive timing. Journal of Experimental Psychology: Human Perception \& Performance, 20, 154-173.

Tresilian, J. R. (1994b). Perceptual and motor processes in interceptive timing. Human Movement Science, 13, 335-373.

Tresilian, J. R. (1995). Study of a servo-control strategy for projectile interception. Quarterly Journal of Experimental Psychology, 48A, 688-715.

Tresilian, J. R. (1997). A revised tau hypothesis: Consideration of Wann's (1996) analyses. Journal of Experimental Psychology: Human Perception \& Performance, 23, 1272-1281.

TRESILIAN, J. R. (2004). Interceptive action: What's time-to-contact got to do with it? In H. Hecht \& G. J. P. Savelsbergh (Eds.), Time-to-contact (pp. 109-140). Amsterdam: Elsevier.

Tresilian, J. R., \& Houseman, J. H. (in press). Systematic variations 
in performance of an interceptive action with changes in the temporal constraints. Quarterly Journal of Experimental Psychology.

Tresilian, J. R., \& Lonergan, A. (2002). Intercepting a moving target: Effects of temporal precision constraints and movement amplitude. Experimental Brain Research, 142, 193-207.

TrESILIAN, J. R., OLIVER, J., \& CARROLL, T. J. (2003). Temporal precision of interceptive action: Differential effects of target size and speed. Experimental Brain Research, 148, 425-438.

Tresilian, J. R., Plooy, A., \& CARroll, T. J. (2004). Constraints on the spatio-temporal accuracy of interceptive action: Effects of target size on hitting a moving target. Experimental Brain Research, 155, 509-526.

Tyldesley, D. A., \& Whiting, H. T. A. (1975). Operational timing. Journal of Human Movement Studies, 1, 172-177.

Valls-Sole, J., Rothwell, J. C., Goulart, F., Cossu, G., \& Munoz, E. (1999). Patterned ballistic movements triggered by a startle in healthy humans. Journal of Physiology, 516, 931-938.

van Donkelaar, P., Lee, R. G., \& Gellman, R. S. (1992). Control strategies in directing the hand to moving targets. Experimental Brain Research, 91, 151-161.

WatTs, R. G., \& BAhILl, A. T. (1990). Keep your eye on the ball: The science and folklore of baseball. New York: Freeman.

Welch, C. M., Banks, S. A., CoOK, F. F., \& Draovitch, P. (1995). Hitting a baseball: A biomechanical description. Journal of Orthopaedic \& Sports Physical Therapy, 22, 193-201.

Williams, T., \& UnderwOOD, J. (1970). The science of hitting. New York: Simon \& Schuster.
Wolpert, D. M., Miall, R. C., \& Kawato, M. (1998). Internal models in the cerebellum. Trends in Cognitive Sciences, 2, 338-347.

ZaAL, F. T. J. M., Bootsma, R. J., \& VAN Wieringen, P. C. W. (1999). Dynamics of reaching for stationary and moving objects: Data and model. Journal of Experimental Psychology: Human Perception \& Performance, 25, 149-161.

\section{NOTES}

1. A role for internally derived information through internal feedback loops (cf. Desmurget \& Grafton, 2000) or proprioceptive information through spinal reflexes (e.g., Feldman \& Levin, 1995) is not at issue.

2. Neuromechanical system is a term derived from Enoka (2002) as a convenient shorthand for referring to the peripheral musculoskeletal system and its spinal control mechanisms.

3. Internal motor noise is often assumed to be multiplicative (e.g., Harris \& Wolpert, 1998; Schmidt et al., 1979) and to have both central and peripheral components.

4. In order to define such an error, it is necessary to choose a location that the person is aiming at. The choice made in the text is somewhat arbitrary but is convenient for illustrative purposes.

(Manuscript received August 7, 2003; revision accepted for publication March 29, 2004.) 